\title{
ÉRIC MATZINGER Étude des solutions surstables de l'équation de Van der Pol
}

Annales de la faculté des sciences de Toulouse $\sigma^{e}$ série, tome 10, $\mathrm{n}^{\mathrm{o}} 4$ (2001), p. 713-744

<http://www.numdam.org/item?id=AFST_2001_6_10_4_713_0>

(C) Université Paul Sabatier, 2001, tous droits réservés.

L'accès aux archives de la revue «Annales de la faculté des sciences de Toulouse » (http://picard.ups-tlse.fr/ annales/) implique l'accord avec les conditions générales d'utilisation (http://www.numdam.org/conditions). Toute utilisation commerciale ou impression systématique est constitutive d'une infraction pénale. Toute copie ou impression de ce fichier doit contenir la présente mention de copyright.

\section{NumDam}

Article numérisé dans le cadre du programme Numérisation de documents anciens mathématiques http://www.numdam.org/ 


\title{
Étude des solutions surstables de l'équation de Van der Pol
}

\author{
ÉRIC MATZINGER ${ }^{(1)}$
}

Résumé. - Il est bien connu que l'équation de Van der Pol forcée $\varepsilon \ddot{u}+\left(u^{2}-1\right) \dot{u}+u=\alpha$ (avec $\dot{u}=\frac{d u}{d t}$ et $\varepsilon \rightarrow 0$ ) admet des solutions particulières (dites «canards 》) pour certaines valeurs de $\alpha(\varepsilon)$, qui correspondent, dans le champ complexe, aux solutions bornées en $\varepsilon$ près de $u=1$. Il existe deux solutions canards distinctes uniques $\left(v^{+}, \alpha^{+}\right),\left(v^{-}, \alpha^{-}\right)$de l'équation transformée $\varepsilon v \frac{d v}{d u}=\left(1-u^{2}\right) v+\alpha-u$ qui restent bornées dans de grands domaines de $\mathbb{C}$ incluant tout un demi-plan contenant 0 et en particulier le point $u=1$. On commence par montrer qu'il existe un développement asymptotique uniforme de la solution $v^{+}$dans des secteurs centrés en -1 tels $\left\{0<\arg (x+1)<2 \pi / 3\right.$, et $\left.|x+1|>\left|X_{l}\right||\varepsilon|^{1 / 3}\right\}$. On en déduira un équivalent de $\alpha^{+}-\alpha^{-}$et des coefficients $a_{n}$ du développement asymptotique $\hat{\alpha}$ des $\alpha$ quand $\varepsilon$ tend vers 0 .

Abstract. - It is well known that the forced Van der Pol equation $\varepsilon \ddot{u}+\left(u^{2}-1\right) \dot{u}+u=\alpha$ (where $\dot{u}=\frac{d u}{d t}$ and $\varepsilon \rightarrow 0$ ) in a singularly perturbed differential equation having exceptional solutions (called canards) for some values of $\alpha(\varepsilon)$. There are exactly two distinct complex canards solutions $\left(v^{+}, \alpha^{+}\right),\left(v^{-}, \alpha^{-}\right)$of the transformed equation $\varepsilon v \frac{d v}{d u}=\left(1-u^{2}\right) v+\alpha-u$, which are bounded in wide open sets of $\mathbb{C}$, including a whole half-plane containing the points $u=0$ and $u=1$. We first will prove the existence of a uniform asymptotic development of the solution $v^{+}$in sectors centered at $u=-1$, like $\{0<\arg (x+1)<2 \pi / 3$, and $\left.|x+1|>\left|X_{1}\right||\varepsilon|^{1 / 3}\right\}$. Explicit asymptotic approximations for $\alpha^{+}-\alpha^{-}$ as $\varepsilon \rightarrow 0$ and, as $n \rightarrow \infty$, of the $a_{n}$ (of the asymptotic serie $\hat{\alpha}=\sum a_{n} \varepsilon^{n}$ corresponding to $\alpha$ ) can then be calculated.

(*) Reçu le 7 mars 2001, accepté le 3 mai 2002

(1) U.F.R. de Mathématique, 7 rue René Descartes, 67084 Strasbourg Cedex. e-mail: Eric.Matzinger@math.u-strasbg.fr 


\section{Introduction}

L'équation de van der Pol [6]

$$
\varepsilon \ddot{u}+\left(u^{2}-1\right) \dot{u}+u=0
$$

est un exemple connu d'équation différentielle dont les solutions présentent des oscillations rapides entre deux périodes de relaxation.

On considèrera ici l'équation de van der Pol «forcée»

$$
\varepsilon \ddot{u}+\left(u^{2}-1\right) \dot{u}+u=\alpha
$$

où $\dot{u}$ désigne la dérivée $\frac{d u}{d t}$, où $\varepsilon$ est un paramètre arbitrairement petit et $\alpha$ un paramètre réel.

Dans toute la suite, on se limitera au cas $\alpha \geqslant 0$ (le cas $\alpha$ négatif lui étant symétrique).

Pour étudier les solutions de cette équation, on se place dans le plan des phases (ou plan de Liénard, [4]), en prenant le système équivalent suivant:

$$
\left\{\begin{aligned}
\varepsilon \dot{u} & =y-\frac{u^{3}}{3}+u \\
\dot{y} & =\alpha-u
\end{aligned}\right.
$$

Une étude sommaire à partir de ce système montre que, pour $\alpha>1$, la trajectoire rejoint rapidement le voisinage de la cubique d'équation $y=$ $u^{3} / 3-u$, puis joint le point $A\left(\alpha, \alpha^{3} / 3-\alpha\right)$ qui est stationnaire. Pour $\alpha<1$, $\alpha$ pas trop proche de 1, la trajectoire finit par contre en un cycle comprenant deux parties stables de la cubique et deux segments horizontaux (cf. figure 1). Une étude locale au voisinage de $A$ montre d'ailleurs qu'il y a une bifurcation de Hopf au voisinage de $\alpha=1$ : le point stationnaire $A$ est attractif si $\alpha<1$, et répulsif si $\alpha>1$ et on a dans ce cas un cycle limite dont la taille est d'ordre $\sqrt{1-\alpha}$ (pour les $\alpha$ très peu différents de 1 ).

Le phénomène dont il est question ici est cependant distinct, quoique très voisin quand on fait varier $\alpha$, de celui de la bifurcation. Il a été découvert quand E. Benoit, J.L. Callot, F. et M. Diener ont cherché à voir, d'abord numériquement, comment on passait (pour $\alpha<1$ ) d'un cycle de petite taille $\sqrt{1-\alpha}$ au cycle beaucoup plus grand de la figure 1 ; d'après la propriété de dépendance continue des solutions (et donc du cycle) par rapport au paramètre $\alpha$ (qui intervient de manière non singulière dans l'équation), on savait qu'il devait exister un régime intermédiaire entre ces deux cycles.

Les premiers résultats obtenus sur ce problème sont les suivants: pour $\varepsilon$ arbitrairement petit fixé, on montre [1] que quand $\alpha$ varie au voisinage de 1 , 


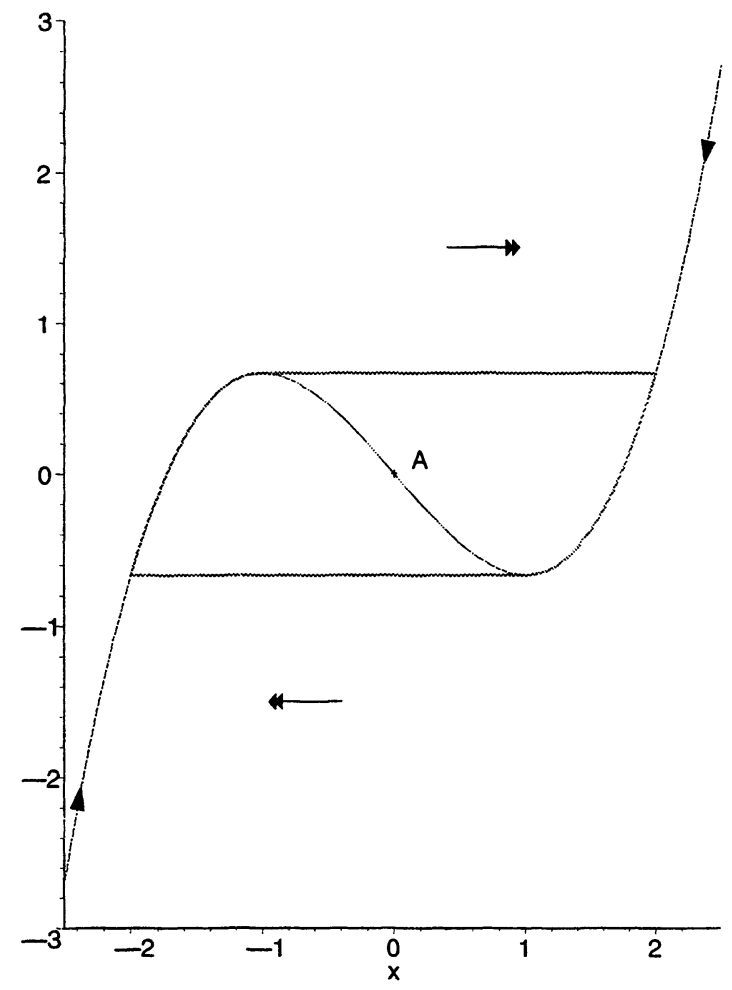

Fig. 1 Cycle pour $\alpha=0$

on peut obtenir un régime intermédiaire entre les deux types de trajectoires cités, dans lequel la trajectoire suit la cubique sur un morceau (aussi grand que l'on veut) de la partie instable de cette courbe; on appelle les solutions correspondantes des «canards». Plus précisément,

1. pour des valeurs $\breve{\alpha}$ voisines de 1 bien choisies, la trajectoire suit la cubique entre ses deux sommets $\left(1,-\frac{2}{3}\right),\left(-1, \frac{2}{3}\right)$ : on a alors ce qu'on appelle des «grands canards»(cf. figure 3 );

2. la trajectoire suit la cubique jusqu'au point d'abscisse $b \in]-1,1[$ si et seulement si $|\alpha-\breve{\alpha}|=\exp \left[-\frac{1}{\varepsilon}\left(R_{1}(b)+o_{\varepsilon}(1)\right)\right]$, où $\breve{\alpha}$ est une des valeurs à grand canard, et $R_{1}(b)=\int_{1}^{b}(\xi-1)(\xi+1)^{2} d \xi$ (voir figure 2);

3. si $\breve{\alpha}$ est une valeur à grand canard, la trajectoire passe aussi dans un voisinage de taille $\varepsilon$ du point $(u=1, y=-2 / 3)$ et la solution du 
Éric Matzinger

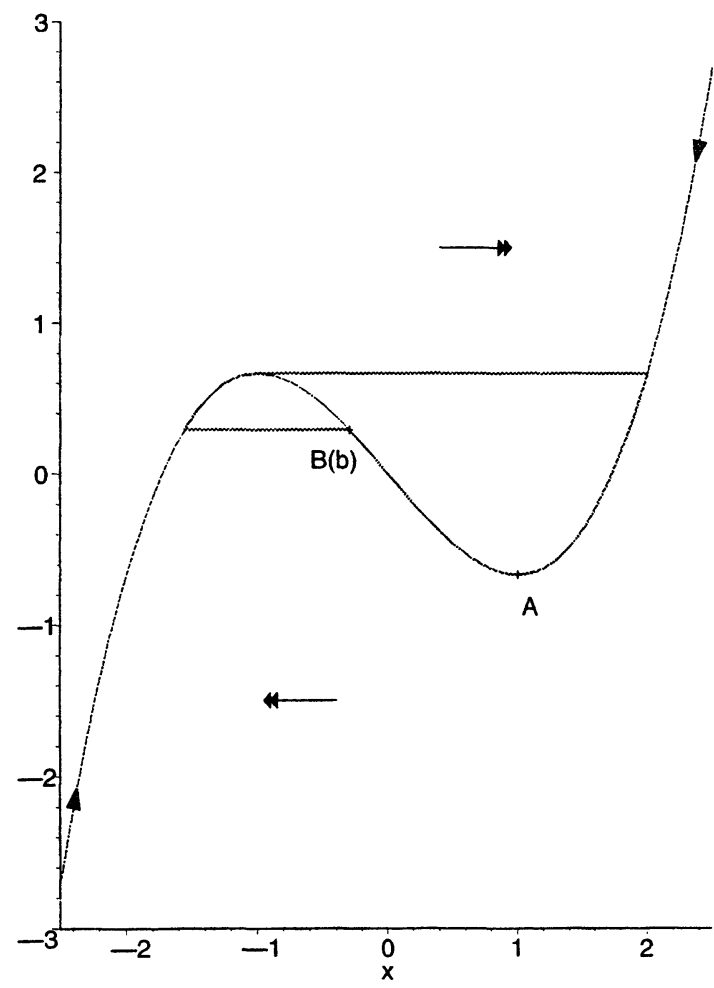

Fig. 2 Canard intermédiaire; B, d'abscisse b, est appelé «col» du canard

système correspondante est donc nécessairement continue en ce point (ou plutôt: bornée en $\varepsilon$ dans un voisinage de ce point).

Ce sont ces solutions particulières qui vont être étudiées. Dans ce but, on effectue le changement de variables suivant, pour se placer au voisinage de la cubique: $y=\frac{u^{3}}{3}-u+\varepsilon v$, ce qui nous mène au système

$$
\left\{\begin{aligned}
\dot{u} & =v \\
\varepsilon \dot{v} & =v\left(1-u^{2}\right)+\alpha-u
\end{aligned}\right.
$$

On élimine la variable temporelle:

$$
\varepsilon v \frac{d v}{d u}=\left(1-u^{2}\right) v+\alpha-u
$$

Comme courbe lente pour cette équation (i.e. pour le paramètre $\varepsilon=0$ ), on trouve la fonction $u \mapsto-\frac{\alpha-u}{1-u^{2}}$, qui n'est continue en 1 que si $\alpha=1$. 


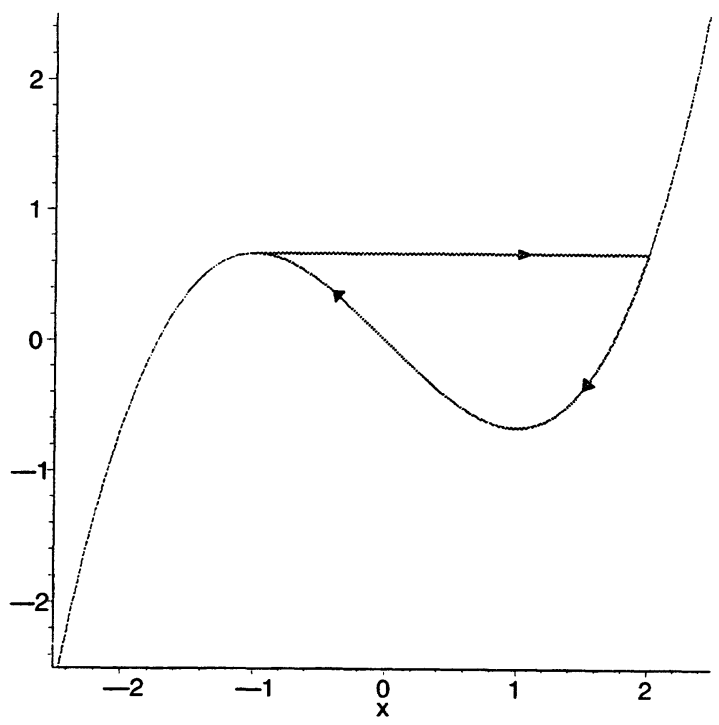

Fig. 3 Grand canard

Dans ce cas, on trouve facilement la solution formelle

$$
\hat{\alpha}=\sum_{n=0}^{\infty} a_{n} \varepsilon^{n} \quad \hat{v}(u)=\sum_{n=0}^{\infty} v_{n}(u) \varepsilon^{n}
$$

avec les formules de récurrence suivantes

$$
\left.\begin{array}{cc}
a_{0}=1 & a_{n+1}=\sum_{j=0}^{n} v_{j}(1) v_{n-j}^{\prime}(1) \\
v_{0}(u)=\frac{-1}{u+1} & v_{n+1}(u)=v_{0}(u) \frac{\sum_{j=0}^{n} v_{j}(u) v_{n-j}^{\prime}(u)-a_{n+1}}{u-1}
\end{array}\right\}
$$

Une étude de cette série, réalisée par M. Canalis-Durand avec les outils de l'analyse Gevrey, a montré qu'elle était divergente, mais Gevrey d'ordre $1[2]$.

L'existence de cette série formelle ne suffit pas, toutefois, à assurer celle d'une solution $v$ de l'équation (E), holomorphe en $u=1$, qui admet $\hat{v}$ comme développement asymptotique uniforme dans tout un voisinage de ce point.

En effet, si on linéarise l'équation différentielle autour de $v_{0}$, en écrivant $v$ sous la forme: $v(u, \varepsilon)=-\frac{1}{u+1}+\frac{w}{u+1}$, et $\alpha=1+a$, on trouve comme 
équation

$$
\begin{aligned}
\varepsilon \frac{d w}{d u}=(u-1)(u+1)^{2} w-(u+1)^{2} a & +\frac{\varepsilon}{u+1}(w-1) \\
& +\frac{w}{1-w}\left((u-1)(u+1)^{2} w-(u+1)^{2} a\right) .
\end{aligned}
$$

Pour une équation ainsi réécrite, on introduit, à partir de l'équation différentielle linéaire homogène, la fonction relief dans le plan des $u$, définie par $R(x)=\Re\left(\int^{x}(u-1)(u+1)^{2} d u\right)$. Les résultats classiques pour une équation singulièrement perturbée sont alors que la solution formelle est effectivement un développement asymptotique uniforme d'une solution holomorphe; mais cela uniquement dans des domaines de points accessibles à partir d'un sommet, c'est à dire de points qu'on peut joindre à partir d'un point fixe appelé sommet par un chemin qui descend toujours le relief $R$. Or $u=1$ est un point col pour ce relief, donc un point tournant pour l'équation: on n'a de développement asymptotique pour une solution que dans des secteurs centrés en ce point, et pas dans un voisinage complet de $u=1$.

Cependant, on a un meilleur résultat pour l'équation de Van der Pol, grâce au fait qu'on peut encore jouer sur le paramètre $\alpha$. Dans [3], en étudiant l'équation dans le champ complexe, il est démontré que:

1. pour $\varepsilon \in \mathbb{R}^{+}$, il existe une unique solution $\left(\alpha^{+}, v^{+}\right)$tel que la solution $v^{+}$existe en particulier, pour n'importe quel $\rho$ réel positif, dans l'ensemble du secteur infini centré non loin de $-1, S(-1+$ $\left.\rho,-\frac{\pi}{6}, \frac{5 \pi}{6}\right)=\{z \in \mathbb{C} / \arg (z+1-\rho) \in]-\frac{\pi}{6}, \frac{5 \pi}{6}[\} ; v^{+}$correspond dans son domaine de définition à la solution formelle $\hat{v}$;

2. pour $\alpha^{-}=\overline{\alpha^{+}}$, on obtient aussi une vraie solution $v^{-}$existant dans un domaine symétrique de celui de $v^{+}$;

3. il existe une solution $(\alpha(\varepsilon), v(u, \varepsilon))$ holomorphe en $\varepsilon$ avec une singularité en $\varepsilon=0$ qui prolonge la solution $\left(\alpha^{+}, v^{+}\right)$dans un large secteur centré en 0 (pour la variable $\varepsilon$ ); cette solution vérifie en plus la propriété: $v^{-}(u, \varepsilon)=v\left(\bar{u}, \varepsilon e^{-2 i \pi}\right), \alpha^{-}(\varepsilon)=\alpha\left(\varepsilon e^{-2 i \pi}\right)$ (i.e. en effectuant un tour complet dans le plan des $\varepsilon$ autour de 0 , en partant de $\varepsilon>0$, on passe de $\alpha^{+}$à $\alpha^{-}$).

4. pour tout $\varepsilon \in \mathbb{C}$ assez petit tel que $|\arg (\varepsilon)|<\frac{\pi}{2}+\delta$, on a

$$
\left|\alpha\left(\varepsilon e^{-2 i \pi}\right)-\alpha(\varepsilon)\right| \leqslant \exp \left(\Re\left(\frac{R_{1}\left(-1+\delta^{\prime}\right)}{\varepsilon}\right)\right)
$$

où $\delta$ et $\delta^{\prime}$ sont des constantes positives, et $R_{1}(x)=\int_{1}^{x}(t-1)(t+1)^{2} d t$. 
L'analyse à partir de cette série formelle ne permet pas cependant de voir ce qui se passe au voisinage de $(-1)$, puisque la courbe lente y a une singularité; alors que le dernier résultat ci-dessus laisse penser qu'on gagnerait en précision si on pouvait remplacer $\delta^{\prime}$ par quelquechose qui tend vers 0 avec $\varepsilon$.

Nous allons regarder spécifiquement ce qui se passe en ce point en introduisant un nouveau changement de variable qui crée une loupe près de $(-1)$ :

$$
u=-1+\varepsilon^{1 / 3} X \quad, \quad v=\varepsilon^{-1 / 3} Y
$$

d'où

$$
(\mathrm{E}) \Longleftrightarrow \quad Y \frac{d Y}{d X}=2 X Y\left(1-\frac{\varepsilon^{1 / 3}}{2} X\right)+\alpha+1-\varepsilon^{1 / 3} X \quad \mathrm{E}^{\prime}
$$

La courbe lente (pour $\varepsilon=0$ et le $\alpha=1$ correspondant) suivra l'équation

$$
Y_{0} \frac{d Y_{0}}{d X}=2 X Y_{0}+2
$$

Nous allons étudier les solutions de (2) bornées à l'infini et montrer que

ThÉORÈme [COROLlaIre 3] .- Il existe une vraie solution $Y^{+}\left(X, \varepsilon^{1 / 3}\right)$ de l'équation différentielle $\left(\mathrm{E}^{\prime}\right)$ qui admet, dans un certain secteur en l'infini, un développement asymptotique uniforme du type $\hat{Y}(X, \varepsilon)=\sum_{n=0}^{\infty} Y_{n}(X) \varepsilon^{n / 3}$.

Comme l'intersection du domaine de $Y^{+}\left(x \varepsilon^{-1 / 3}, \varepsilon^{1 / 3}\right)$ avec celui de $v^{+}(x, \varepsilon)$ est non vide et qu'elles sont égales dans cette intersection, par unicité, $Y^{+}$est le prolongement analytique de $z^{+}$au voisinage de $u=-1$. L'existence de cette solution permettra de trouver les résultats suivants, dans les paragraphes 4 et 5 respectivement:

Résultat 1 Un équivalent exact de la différence $\alpha^{+}-\alpha^{-}$quand $\varepsilon$ tend vers 0 est

$$
\alpha^{+}-\alpha^{-} \sim i \frac{e^{-\frac{4}{3 \varepsilon}}}{\sqrt{\varepsilon}} \frac{8 \sqrt{2}}{\sqrt{\pi} e^{4 / 3}} .
$$

Résultat 2 Les coefficients $a_{n}$ de la série $\hat{\alpha}$ ci-dessus sont équivalents, quand $n \rightarrow \infty$, ̀̀

$$
\begin{gathered}
a_{n} \sim \frac{-4 \sqrt{3}}{\pi e^{4 / 3}}\left(\frac{3 n}{4 e}\right)^{n} . \\
-719-
\end{gathered}
$$




\section{2. Étude de la courbe lente}

Il est possible de donner, d'une certaine manière, une solution exacte de l'équation de la courbe lente (2) qui vérifie les conditions aux limites voulues. Plus exactement, nous allons démontrer le théorème suivant:

THÉORÈme 1. - Il existe des solutions de l'équation différentielle

$$
Y_{0} \frac{d Y_{0}}{d X}=2 X Y_{0}+2
$$

qui tendent vers 0 en l'infini et dont les domaines d'existence contiennent des secteurs ouverts d'ouverture au moins $\frac{2 \pi}{3}$ en l'infini. En particulier, il $y$ en a une qui est holomorphe dans un secteur $S\left(\infty, \varrho,-\frac{\pi}{6}, \frac{5 \pi}{6}\right)$, et une autre dans un domaine symétrique contenant $S\left(\infty, \varrho,-\frac{5 \pi}{6}, \frac{\pi}{6}\right)$. Ces deux solutions peuvent être prolongées jusqu'en 0.

Pour démontrer ce théorème, on commence par effectuer le changement de variable suivant:

$$
Y_{0}(X)=X^{2}+z(X) \Longrightarrow \quad(2) \quad z^{\prime}(X)=\frac{2}{X^{2}+z(X)}
$$

On regarde ensuite la fonction réciproque de la fonction $X \mapsto z(X):$ on considère en fait $z \mapsto X(z)$; on sait, d'après les théorèmes sur les fonctions implicites que si $z^{\prime}$ ne s'annule pas, la dérivée de $X$ s'écrira:

$$
\frac{d X(z)}{d z}=\frac{X^{2}(z)+z}{2} \text {. }
$$

On pose ensuite

$$
X=-2 \frac{u^{\prime}}{u}, \quad \text { d'où } \quad u^{\prime \prime}+\frac{1}{4} u z=0,
$$

qui est une équation d'Airy.

Les solutions de l'équation d'Airy peuvent toutes s'écrire (par exemple) à partir des fonctions d'Airy $\mathrm{Ai}$ et $\mathrm{Bi}$.

On note

$$
\mu=\sqrt[3]{\frac{1}{4}}, \quad j=e^{2 i \pi / 3}
$$

Une des solutions correspondra par exemple à $u=A \mathrm{Ai}\left(-\mu j^{2} z\right)$ :

$$
x=2 \mu j^{2} \frac{A i^{\prime}\left(-\mu j^{2} z\right)}{A i\left(-\mu j^{2} z\right)}
$$


Or, les résultats connus (voir [5]) sur Ai sont:

$$
\begin{aligned}
\operatorname{Ai}(z) & =\frac{e^{-\xi}}{2 \sqrt{\pi} z^{1 / 4}}\left(1+\mathcal{O}\left(\frac{1}{\xi}\right)\right) \\
\operatorname{Ai}^{\prime}(z) & =\frac{-e^{-\xi} z^{1 / 4}}{2 \sqrt{\pi}}\left(1+\mathcal{O}\left(\frac{1}{\xi}\right)\right)
\end{aligned}
$$

où $\xi=\frac{2}{3} z^{3 / 2}$ (en prenant la valeur principale de la racine), ceci pour

$$
-\pi+\delta \leqslant \arg z \leqslant \pi-\delta \quad(\delta>0) .
$$

Comme $\arg \left(-\mu j^{2} z\right)=\arg (z)+\pi / 3$, on s'intéresse aux $z$ tels que $-\frac{4 \pi}{3}+\delta \leqslant$ $\arg z \leqslant \frac{2 \pi}{3}+\delta$, et on voit que dans ce cas

$$
\begin{aligned}
X^{2}(z) & =4 \frac{u^{\prime 2}(z)}{u^{2}(z)}=4 \mu^{2} j^{4}\left(-\mu j^{2} z\right)\left(1+\mathcal{O}\left(\frac{1}{\xi}\right)\right) \\
& =-z+\mathcal{O}\left(\frac{1}{\sqrt{|z|}}\right) .
\end{aligned}
$$

Cette dernière égalité permet d'affirmer, en utilisant le théorème d'inversion locale, que la fonction $z \mapsto X(z)$ a un inverse quand $z$ est dans un voisinage de l'infini dans le secteur proposé, soit la fonction $X \mapsto z(X)$. pour

Et alors $Y_{0}(X)=X^{2}+z(X)=\mathcal{O}(1 / \sqrt{|z|})$ tend vers 0 quand $X \rightarrow \infty$

$$
-\frac{\pi}{6}+\delta^{\prime} \leqslant \arg X \leqslant \frac{5 \pi}{6}-\delta^{\prime}
$$

On obtient bien une solution $Y_{0}$ bornée sur un large secteur de sommet 0 . D'après l'équation différentielle (2), $Y_{0}$ ne pourrait tendre vers l'infini que quand $X$ tend vers l'infini. En effet, supposons qu'il existe une suite de points $\left(X_{n}\right)$ convergeant vers un point fini $X_{\infty}$, telle que $Y_{0}\left(X_{n}\right)$ soit une suite non bornée. Alors la fonction $u_{0}=1 / Y_{0}$ vérifie l'équation

$$
\frac{d u_{0}}{d X}=-2 X u_{0}^{2}-2 u_{0}^{3}
$$

et elle est telle que $u_{0}\left(X_{n}\right) \rightarrow 0$. Comme $u_{0}$ n'a pas de singularité en $X_{\infty}$, et que d'après le théorème de Painlevé elle vérifie $u_{0}\left(X_{\infty}\right)=0$, il s'agit de l'unique solution de son équation qui s'annule en $X_{\infty}$, donc de la fonction nulle. Ce qui est absurde. Les seules singularités de $Y_{0}$ sont donc des zéros de $Y_{0}$.

L'équation différentielle nous indique aussi que

$$
\begin{gathered}
Y_{0} \underset{X \rightarrow \infty}{\sim}-\frac{1}{X}: \\
-721-
\end{gathered}
$$


comme $Y_{0}$ est bornée dans un domaine comprenant un secteur en l'infini, d'après la formule de Cauchy, $Y_{0}^{\prime}$ est bornée elle aussi à l'intérieur de ce domaine. Donc quand $X$ devient grand, on a nécessairement que $2 X Y_{0}+2 \rightarrow$ 0 . D'où le résultat.

Si on veut aller plus loin dans le développement de $Y_{0}$, il suffit de décomposer la fonction $Y_{0}=-1 / X+\tilde{Y}$. La fonction $\tilde{Y}$ est solution (bornée quand $X$ devient grand dans le bon secteur) de

$$
\tilde{Y^{\prime}} \tilde{Y}=2 X \tilde{Y}+\frac{1}{X^{3}}-\frac{\tilde{Y}}{X^{2}}+\frac{\tilde{Y}^{\prime}}{X} .
$$

$\tilde{Y}=o(1 / X)$; avec Cauchy, on en déduit donc que $\tilde{Y}^{\prime}$ est au plus un $o\left(1 / X^{2}\right)$. Dans l'équation différentielle, les deux termes les plus importants sont cette fois $2 X \tilde{Y}$ et $1 / X^{3}$, donc $\tilde{Y} \sim-1 / 2 X^{4}$.

Au total,

$$
Y_{0}=-\frac{1}{X}-\frac{1}{2 X^{4}}+o\left(\frac{1}{X^{4}}\right)
$$

On peut montrer que la solution proposée existe aussi sur l'ensemble du demi-axe $\left\{z \in \mathbb{C} / \arg z=-\frac{\pi}{3}\right\}$.

En effet, pour $z=-j t \quad\left(t \in \mathrm{R}^{+}\right)$,

$$
\begin{gathered}
X(z)=2 \mu j^{2} \frac{\mathrm{Ai}^{\prime}(\mu t)}{\mathrm{Ai}(\mu t)} \quad \arg X(z)=\arg j^{2}+\pi-0=\frac{\pi}{3} \\
\frac{d X}{d t}=\frac{d X}{d z} \frac{d z}{d t}=\frac{X^{2}(z)+z}{2}(-j)=-\frac{j^{2}}{2}\left(4 \mu^{2} \frac{\mathrm{Ai}^{2}(\mu t)}{\mathrm{Ai}^{2}(\mu t)}-t\right) .
\end{gathered}
$$

Le calcul de la dérivée de $4 \mu^{2} \mathrm{Ai}^{2}(\mu t)-t \mathrm{Ai}^{2}(\mu t)$, montre qu'elle est nulle; cette fonction est par conséquent une fonction constante, et réelle, donc $\frac{-1}{j^{2}} \frac{d x}{d t}$ est une fonction réelle qui garde un signe constant (puisqu'on l'obtient de la précédente en divisant $\operatorname{par} \mathrm{Ai}^{2}(\mu t) \in \mathbb{R}^{+}$).

D'où l'on peut déduire que $t \mapsto \frac{x(t)}{-j^{2}}$ est réelle et uniforme sur $\mathbb{R}^{+}$, donc inversible.

On peut bien sûr prolonger analytiquement cette fonction sur tout domaine simplement connexe ne contenant pas de singularité de $Y_{0}$ (les seules singularités possibles étant les points où $Y_{0}$ s'annule). Cela semble être le cas par exemple (quand on fait des simulations numériques) du quart de plan $(\Re z \geqslant 0, \Im z \geqslant 0)$. On sait en tous cas que $Y_{0}$ existe sur une partie de l'axe réel, à partir d'un réel $X_{l} \geqslant 0$ jusqu'au voisinage de l'infini. 
3. Existence d'une solution de $\left(\mathrm{E}^{\prime}\right), Y(X, \varepsilon)$, qui est voisine de $Y_{0}$

Nous utiliserons un théorème de point fixe pour montrer que certaines solutions de $\left(\mathrm{E}^{\prime}\right)$ tendent vers $Y_{0}$ quand $\varepsilon$ tend vers 0 .

$$
\left(\mathrm{E}^{\prime}\right) \quad Y Y^{\prime}=2 X Y\left(1-\frac{\varepsilon^{\prime}}{2} X\right)+\alpha+1-\varepsilon^{\prime} X, \quad \text { avec } \varepsilon^{\prime}=\varepsilon^{1 / 3}
$$

On écrit $Y$ sous la forme $Y=Y_{0}+\varepsilon^{\prime} Z$. On rappelle (2): $Y_{0} Y_{0}^{\prime}=2 X Y_{0}+2$.

Alors

$$
\begin{aligned}
Y_{0} Y_{0}^{\prime}+\varepsilon^{\prime} Z^{\prime}\left(Y_{0}+\varepsilon^{\prime} Z\right)+\varepsilon^{\prime} Z\left(2 X+\frac{2}{Y_{0}}\right) \\
=2 X Y_{0}-\varepsilon^{\prime} X^{2} Y_{0}+\varepsilon^{\prime} Z X\left(2-\varepsilon^{\prime} X\right)+\alpha-1+2-\varepsilon^{\prime} X \\
\Longleftrightarrow Z^{\prime}=-X^{2}+\frac{a / \varepsilon^{\prime}-X-2 Z / Y_{0}}{Y_{0}+\varepsilon^{\prime} Z} \\
\text { avec } \quad a=\alpha-1=\mathcal{O}(\varepsilon)=o\left(\varepsilon^{\prime}\right) .
\end{aligned}
$$

Après linéarisation de cette équation différentielle, on obtient

$$
\begin{gathered}
Z^{\prime}=-X^{2}+u_{0}\left(\frac{a}{\varepsilon^{\prime}}-X\right)-Z\left(u_{0}^{2}\left(2+a-\varepsilon^{\prime} X\right)\right)+\varepsilon^{\prime} h\left(Z, X, \varepsilon^{\prime}\right) \\
\text { où } \quad u_{0}(X)=\frac{1}{Y_{0}(X)}=-X+\frac{1}{2 X^{2}}+o\left(\frac{1}{X^{2}}\right) \\
\text { et } \quad h\left(Z, X, \varepsilon^{\prime}\right)=Z^{2} u_{0}^{3} \frac{2+a-\varepsilon^{\prime} X}{1+\varepsilon^{\prime} u_{0} Z} .
\end{gathered}
$$

On introduit les fonctions

$$
\begin{aligned}
F(x) & =\int^{x}-u_{0}^{2}(t)\left(2+a-\varepsilon^{\prime} t\right) d t, \\
\operatorname{et} R(x) & =\Re(F(x)) .
\end{aligned}
$$

On désignera par $\gamma_{X}$ un chemin dans le plan complexe partant d'un point (éventuellement infini) à déterminer et allant jusqu'à $X$.

En intégrant l'équation différentielle avec la méthode de variation de la constante, on voit que $Z$ satisfait à l'égalité:

$$
Z(X)=\int_{\gamma_{X}} e^{F(X)-F(t)}\left(-t^{2}+u_{0}(t)\left(\frac{a}{\varepsilon^{\prime}}-t\right)+\varepsilon^{\prime} h\left(Z, t, \varepsilon^{\prime}\right)\right) d t .
$$


On souhaite montrer qu'on peut appliquer un théorème de point fixe, pour montrer qu'il existe une et une seule solution à l'équation $\left(E^{\prime}\right)$ qui tend vers $Y_{0}$ quand $\varepsilon$ tend vers 0 . On se place pour cela dans un domaine ouvert $\mathcal{D}$ (qui sera précisé par après) et on considère deux solutions notées $Y_{1}$ et $Y_{2}$ arbitrairement proches de $Y_{0}$.

Soit alors $h_{i}=h\left(Y_{i}, t, \varepsilon^{\prime}\right)$, pour $i=1,2$.

On trouve

$$
h_{1}-h_{2}=\left[u_{0}(t)\left(2+a-\varepsilon^{\prime} t\right)\right]\left[u_{0}(t)\left(Y_{1}-Y_{2}\right)\right] \frac{\left(Y_{1}+Y_{2}\right) u_{0}+\varepsilon^{\prime} Y_{1} Y_{2} u_{0}^{2}}{\left(1+\varepsilon^{\prime} Y_{1} u_{0}\right)\left(1+\varepsilon^{\prime} Y_{2} u_{0}\right)}
$$

On est donc amené à introduire les espaces fonctionnels suivants:

$$
\begin{aligned}
& \mathfrak{H}=\left\{\text { fonctions holomorphes } h \operatorname{sur} \mathcal{D} / \sup _{t \in \mathcal{D}} \frac{|h(t)|}{\left|u_{0}(t)\right| \cdot\left|2+a-\varepsilon^{\prime} t\right|} \text { soit fini }\right\} \\
& \mathfrak{Y}=\left\{\text { fonctions holomorphes } Y \operatorname{sur} \mathcal{D} / \sup _{t \in \mathcal{D}}|Y(t)| \cdot\left|u_{0}(t)\right| \text { soit fini }\right\}
\end{aligned}
$$

que l'on munit des normes correspondantes $\|h\|_{\mathfrak{H}}$ et $\|Y\|_{\mathfrak{Y}}$ respectivement.

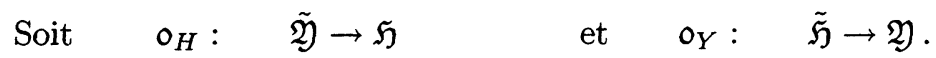

$$
\begin{aligned}
& Y \mapsto h\left(Y, t, \varepsilon^{\prime}\right) \quad h \mapsto Z(X)
\end{aligned}
$$

Ces deux opérateurs sont bien définis au moins sur des sous-ensembles $\tilde{\mathfrak{H}}$ et $\tilde{\mathfrak{Y}}$ des espaces $\mathfrak{H}$ et $\mathfrak{Y}$ (ceci sera reprécisé plus loin).

Nous allons montrer que l'opérateur $o_{Y} o_{H}$ est une contraction sur ces ensembles.

$$
\left\|h_{1}-h_{2}\right\|_{\mathfrak{H}} \leqslant\left\|Y_{1}-Y_{2}\right\|_{\mathfrak{Y}} \frac{\left\|Y_{1}\right\|_{\mathfrak{Y}}+\left\|Y_{2}\right\|_{\mathfrak{Y}}+\left|\varepsilon^{\prime}\right| \cdot\left\|Y_{1}\right\|_{\mathfrak{Y}}\left\|Y_{2}\right\|_{\mathfrak{Y}}}{\left(1-\left|\varepsilon^{\prime}\right| \cdot\left\|Y_{1}\right\|_{\mathfrak{Y}}\right)\left(1-\left|\varepsilon^{\prime}\right| \cdot\left\|Y_{2}\right\|_{\mathfrak{Y}}\right)}
$$

donc si $\left\|Y_{1}\right\|_{\mathfrak{Y}}$ et $\left\|Y_{2}\right\|_{\mathfrak{Y}}$ sont majorées par $\delta$, et pour $\varepsilon^{\prime}<1 / \delta$, on a

$$
\left\|h_{1}-h_{2}\right\|_{\mathfrak{H}} \leqslant\left\|Y_{1}-Y_{2}\right\|_{\mathfrak{Y}} \frac{2 \delta+\left|\varepsilon^{\prime}\right| \delta^{2}}{\left(1-\left|\varepsilon^{\prime}\right| \delta\right)^{2}} .
$$

Par ailleurs, si $Y_{k}\left(X, \varepsilon^{\prime}\right)=\mathrm{oo}_{Y}\left(h_{k}\left(X, \varepsilon^{\prime}\right)\right)$,

$$
Y_{1}-Y_{2}=\int_{\gamma_{X}} e^{F(X)-F(t)} \varepsilon^{\prime}\left(h_{1}-h_{2}\right) d t
$$


d'où

$$
\begin{aligned}
& \left\|Y_{1}-Y_{2}\right\|_{\mathscr{Y}} \leqslant \sup _{X \in \mathcal{D}}\left|u_{0}(X) \| \varepsilon^{\prime}\right| \int_{\gamma_{X}} e^{R(X)-R(t)}\left|h_{1}-h_{2}\right| d t \\
& \quad \leqslant\left|\varepsilon^{\prime}\right| .\left\|h_{1}-h_{2}\right\|_{\mathcal{H}} \sup _{X \in \mathcal{D}} e^{R(X)} \int_{\gamma_{X}} e^{-R(t)}\left|u_{0}^{2}(t)\left(2+a-\varepsilon^{\prime} t\right)\right|\left|\frac{u_{0}(X)}{u_{0}(t)}\right| d t
\end{aligned}
$$

Choisissons un $X$ tel que: pour tout $X^{\prime}$ tel que $\left|X^{\prime}\right| \geqslant|X|, u_{0}\left(X^{\prime}\right)$ est peu différent de $-X^{\prime}$. Plus précisément, on va choisir $\gamma_{X}$ et $X$ tels que:

1. $\gamma_{X}(t) \in \mathcal{D}$ pour tout $t$.

2. $\gamma_{X}$ va de l'infini à $X$ avec $\gamma_{X}(t)=e^{i \theta} t$, où $t$ est un réel allant de $+\infty$ à $|X|$, et $\theta=\arg X$.

Alors, pour $\theta$ dans l'intervalle $] \frac{3 \pi}{8}+\delta, \frac{\pi}{2}-\delta[$,

$$
\begin{aligned}
F(X)-F(t) & =\int_{|X| e^{i \theta}}^{|t| e^{i \theta}}-u_{0}^{2}(\phi)\left(2+a-\varepsilon^{\prime} \phi\right) d \phi \quad t=|t| e^{i \theta} \\
& =\int_{|X|}^{|t|}(2+a)\left(-\phi^{2}\right) d \phi+\varepsilon^{\prime} \int_{|X|}^{|t|} \phi^{3} d \phi+o\left(|t|^{2}\right) \\
& =\frac{2+a}{3} e^{i(3 \theta-\pi)}|t|^{3}+\frac{\varepsilon^{\prime}}{4} e^{4 i \theta}|t|^{4}+o\left(|t|^{2}\right)
\end{aligned}
$$

La partie réelle de cette dernière expression est positive, (pour $|t|$ assez grand donné indépendamment de $\left|\varepsilon^{\prime}\right|$, pour tout $\arg \left(\varepsilon^{\prime}\right)$ assez voisin de 0 ) et croissante avec $|t|$. Donc si on choisit un $X$ assez grand, il existe un $\mu>0$ tel que

$$
\mu\left|\frac{d}{d t} R\left(\gamma_{X}(t)\right)\right| \geqslant\left|F^{\prime}\left(\gamma_{X}(t)\right)\right|, \text { pour tout } t,
$$

d'où on déduit

$$
\begin{aligned}
\left\|Y_{1}-Y_{2}\right\|_{\mathfrak{Y}} & \leqslant\left|\varepsilon^{\prime}\right| \cdot\left\|h_{1}-h_{2}\right\|_{\mathcal{H}} \sup _{X \in \mathcal{D}} e^{R(X)} \int_{\gamma_{X}} e^{-R(t)} \mu\left|\frac{d}{d t} R\left(\gamma_{X}(t)\right)\right| d t \\
\left\|Y_{1}-Y_{2}\right\|_{\mathfrak{Y}} & \leqslant\left|\varepsilon^{\prime}\right| \cdot \mu \cdot\left\|h_{1}-h_{2}\right\|_{\mathcal{H}}
\end{aligned}
$$

Nous pouvons maintenant préciser le domaine $\mathcal{D}$ :

- $\mathcal{D} \subset\left(\right.$ Domaine de $\left.Y_{0}\right)$ (pour que $u_{0}$ soit définie). 
- Tout point $X \in \mathcal{D}$ peut être joint de l'infini par un chemin $\gamma_{X}$ vérifiant (8).

- $\left(2+a-\varepsilon^{\prime} t\right)$ ne s'annule pas pour $t$ dans ce domaine.

Le domaine $\mathcal{D}$ n'est pas vide: avec les conditions données, on peut prendre par exemple pour $\mathcal{D}$ un secteur $S\left(\infty, 2\left|X_{f}\right|, \frac{3 \pi}{8}+\delta, \frac{\pi}{2}-\delta\right)$.

Alors, pour $\varepsilon$ assez petit, les inégalités (7) et (9) signifient que $o_{Y} 0_{H}$ peut être un opérateur contractant.

Il ne reste qu'à vérifier qu'on a bien $o_{Y}(\mathfrak{H}) \subset \mathfrak{Y}$ et que $o_{H}(\mathfrak{y}) \subset \mathfrak{H}$ où $\mathfrak{y}$ est une boule ouverte de $\mathfrak{Y}$.

Si $Y \in \mathfrak{Y}$ et $\|Y\|_{\mathfrak{Y}}<\frac{1}{2 \varepsilon^{\prime}}$ (on a là la description de l'ensemble $\mathfrak{y}$ ), alors

$$
\begin{aligned}
\mathfrak{o}_{H}(Y)=h\left(Y, X, \varepsilon^{\prime}\right) & =u_{0}(X)\left(Y(X) u_{0}(X)\right)^{2} \frac{2+a-\varepsilon^{\prime} X}{1+\varepsilon^{\prime} u_{0}(X) Y(X)} \\
\left\|h\left(Y, X, \varepsilon^{\prime}\right)\right\|_{\mathcal{H}} & \leqslant \sup _{X \in \mathcal{D}}\left|\frac{\left(y u_{0}\right)^{2}}{1+\varepsilon^{\prime} u_{0} y}\right|
\end{aligned}
$$

qui est bien borné, et à $Y$ fixé est aussi borné pour tout $\varepsilon^{\prime}$ assez petit, puisque $\left|u_{0} Y\right|$ est borné pour $X \in \mathcal{D}$ quand $Y \in \mathfrak{Y}$.

Si $h \in \mathfrak{H}$,

$$
\begin{aligned}
\mathfrak{o}_{Y}(h) & =Z\left(h, X, \varepsilon^{\prime}\right) \\
& =\int_{\gamma_{X}} e^{F(X)-F(t)}\left(-t^{2}+u_{0}(t)\left(\frac{a}{\varepsilon^{\prime}}-t\right)+\varepsilon^{\prime} h\left(t, \varepsilon^{\prime}\right)\right) d t \\
& =Z\left(0, X, \varepsilon^{\prime}\right)+\left(Z\left(h, X, \varepsilon^{\prime}\right)-Z\left(0, X, \varepsilon^{\prime}\right)\right)
\end{aligned}
$$

La différence dans la parenthèse ne posant pas de problèmes vu ce qui a été montré ci-dessus (la fonction nulle est clairement dans l'espace $\mathfrak{H}$ ), on regarde uniquement le premier terme.

$$
Z\left(0, X, \varepsilon^{\prime}\right)=\int_{\gamma_{X}} e^{F(X)-F(t)}\left(-t^{2}+u_{0}(t)\left(\frac{a}{\varepsilon^{\prime}}-t\right)\right) d t
$$

On retrouve quelque chose d'analogue à la démonstration concernant la différence des fonctions $Y_{1}-Y_{2}$.

$\left\|Z\left(0, X, \varepsilon^{\prime}\right)\right\|_{\mathfrak{Y}} \leqslant \frac{1}{\left|\varepsilon^{\prime}\right|} \sup _{X \in \mathcal{D}}\left|u_{0}(X)\right| \int_{\gamma_{X}} e^{R(X)-R(t)}\left|-t^{2} \varepsilon^{\prime}+u_{0}(t)\left(a-\varepsilon^{\prime} t\right)\right| d t$ 


$$
\begin{aligned}
& \leqslant \frac{1}{\left|\varepsilon^{\prime}\right|} \cdot \sup _{X \in \mathcal{D}} e^{R(X)} \int_{\gamma_{X}} e^{-R(t)}\left|-t^{2} u_{0} \varepsilon^{\prime}+u_{0}^{2}(t)\left(a-\varepsilon^{\prime} t\right)\right|\left|\frac{u_{0}(X)}{u_{0}(t)}\right| d t \\
& \leqslant\left\|-u_{0} \varepsilon^{\prime} t\left(u_{0}(t)+t\right)+a u_{0}^{2}(t)\right\|_{\mathcal{H}} \sup _{X \in \mathcal{D}} e^{R(X)} \int_{\gamma_{X}} e^{-R(t)} \mu\left|\frac{d}{d t} R\left(\gamma_{X}(t)\right)\right| d t
\end{aligned}
$$

La fonction $-u_{0} \varepsilon^{\prime} t\left(u_{0}(t)+t\right)+a u_{0}^{2}(t)$ est bien dans $\mathfrak{H}: u_{0}(t)=-t+o(1 / t)$, donc la fonction est majorée par $\left|u_{0}(t)\left(1+|a t|+\left|\varepsilon^{\prime}\right|\right)\right|<\left|u_{0}(t)\left(2+a-\varepsilon^{\prime} t\right)\right|$ pour tout $\varepsilon$ assez petit.

$$
\left\|Z\left(0, X, \varepsilon^{\prime}\right)\right\|_{\mathfrak{Y}} \text { existe bien, } Z\left(0, X, \varepsilon^{\prime}\right) \in \mathfrak{Y} .
$$

On peut donc appliquer le théorème du point fixe, ce qui nous donne l'existence et l'unicité de la solution de $(5), Z\left(X, \varepsilon^{\prime}\right)$, dans l'espace $\mathfrak{Y}$ : cette fonction est holomorphe et tend vers 0 quand $X \rightarrow \infty$, pour tout $\varepsilon^{\prime}$.

On en déduit:

THÉORÈME 2. - Il existe une solution $Y_{\varepsilon}$ de $\left(E^{\prime}\right)$ sur un domaine $\mathcal{D}$ contenant un secteur ouvert centré en l'infini (de taille indépendante de $\left.\left|\varepsilon^{\prime}\right|\right)$ d'ouverture un peu inférieure à $\pi / 8 . Y_{\varepsilon}$ tend vers 0 en l'infini et elle est telle que $Y_{\varepsilon} \underset{\varepsilon \rightarrow 0}{\longrightarrow} Y_{0}$ uniformément.

Ce dòmaine $\mathcal{D}$ peut même être encore étendu.

Montrons qu'on peut, en effet, prendre un domaine $\mathcal{D}$ plus grand que celui proposé. Comme l'équation ( $\left.E^{\prime}\right)$ n'est pas singulière, les chemins $\gamma_{X}$ peuvent être rallongés d'une distance arbitraire (mais finie) à partir de $X_{f}$ (par exemple, on peut aller jusqu'à 0 , ou $X_{l}$ ); l'intégrale

$$
\int_{\gamma_{X}} e^{R(x)-R(t)}\left|u_{0}^{2}(t)\left(2+a-\varepsilon^{\prime} t\right)\right|\left|\frac{u_{0}(X)}{u_{0}(t)}\right| d t
$$

restera effectivement bornée si on évite les singularités de $Y_{0}$, puisque toutes les fonctions qu'elle contient sont holomorphes et donc bornées sur tout compact inclus dans leurs domaines de définition. Et puisqu'il n'y a pas dans cette intégrale de singularité en $\varepsilon$, on pourra choisir des bornes indépendantes de $\varepsilon$.

On peut même étendre $\mathcal{D}$ sur une partie de l'axe réel, et ce jusque $|\varepsilon|^{-1 / 3}$ par exemple. En effet, supposons que l'on ait déjà prolongé $\gamma_{X}$ de $X$ à $X_{l}$, puis éventuellement à $X_{0} \in \mathrm{R}^{+}, X_{0}$ assez grand.

Alors

$$
\begin{gathered}
R(x)=\text { Cste }+\int_{X_{0}}^{x}-t^{2}\left(2+a-\varepsilon^{\prime} t\right) d t+o\left(x^{2}\right) \\
-727-
\end{gathered}
$$




$$
\frac{d}{d t} R(t)=-t^{2}\left(2+a-\varepsilon^{\prime} t\right)+o(t) \in \mathrm{R}^{-} \operatorname{si} t \leqslant \frac{1}{\varepsilon^{\prime}}
$$

(pour $\varepsilon^{\prime}$ assez petit, $a$ étant de l'ordre de ${\varepsilon^{\prime}}^{3}$ )

$$
\begin{aligned}
\left\|Y_{1}-Y_{2}\right\|_{\mathfrak{Y}} & \leqslant\left|\varepsilon^{\prime}\right| .\left\|h_{1}-h_{2}\right\|_{\mathcal{H}} \sup _{x} e^{R(x)} \int_{0}^{1 / \varepsilon^{\prime}} e^{-R(t)}\left(-\frac{d}{d t} R(t)\right) d t \\
& \leqslant\left|\varepsilon^{\prime}\right| .\left\|h_{1}-h_{2}\right\|_{\mathfrak{H}} \times \text { Cste }
\end{aligned}
$$

ce qui implique une convergence uniforme sur $\left[X_{l}, \varepsilon^{-1 / 3}\right]$, puisque l'inégalité (7) reste bien sûr vérifiée tant que l'on ne s'approche pas des singularités où des zéros de la fonction $u_{0}$.

Cette solution $Y_{\varepsilon}$ de $\left(\mathrm{E}^{\prime}\right)$ correspond à une solution $v$ de $(\mathrm{E})$ qui existe donc, pour $\varepsilon>0$, au moins jusqu'en $2\left|X_{f}\right| e^{i 7 \pi / 16} \times \varepsilon^{1 / 3}$, sur le segment $\left[-1+X_{l} \varepsilon^{1 / 3}, 0\right]$ et qui coïncide au voisinage de l'infini avec la solution $y$ étudiée dans [3]: ce sont des prolongements holomorphes l'une de l'autre.

Cette solution $Y_{\varepsilon}$ admet un développement asymptotique uniforme:

Corollaire 3. - Il existe une suite de fonctions $Y_{n}(X)$ holomorphes en $X$ et tendant vers 0 quand $X$ tend vers l'infini telle que, pour tout $N \in \mathbb{N}$, et $X \in \mathcal{D}$,

$$
\left|Y_{\varepsilon}\left(X, \varepsilon^{\prime}\right)-\sum_{n=0}^{N} Y_{n}(x) \varepsilon^{\prime n}\right| \leqslant \varepsilon^{\prime N+1}\left|R_{N}(X)\right|,
$$

où la fonction bornée $R_{N}$ vérifie en outre

$$
\lim _{X \rightarrow \infty} R_{N}(X)=0
$$

Le cas $N=0$ correspond au théorème précédent. On va démontrer le cas général par récurrence, à partir du cas $N=1$. On reprend l'équation 5 :

$$
Z^{\prime}=-X^{2}+u_{0}\left(\frac{a}{\varepsilon^{\prime}}-X\right)-Z\left(u_{0}^{2}\left(2+a-\varepsilon^{\prime} X\right)\right)+\varepsilon^{\prime} h\left(Z, X, \varepsilon^{\prime}\right) .
$$

On a montré que $Z\left(X, \varepsilon^{\prime}\right)$ était une fonction qui existait, dans l'espace $\mathfrak{Y}$, et que sa norme $\|Z\|_{\mathfrak{Y}}$ était bornée indépendamment de $\varepsilon^{\prime}$.

On définit $Y_{1}$ comme solution de l'équation différentielle ci-dessus avec $\varepsilon^{\prime}=0$ :

$$
Y_{1}^{\prime}(X)=-X^{2}-X u_{0}(X)-2 Y_{1}(X) u_{0}^{2}(X),
$$

bornée dans $\mathcal{D}$. Dans le secteur $[3 \pi / 8+\delta, \pi / 2-\delta]$ considéré, le coefficient du terme homogène, $-u_{0}^{2}(X)$, a une partie réelle qui tend vers $+\infty$. Il existe 
donc une et une solution $Y_{1}$ de l'équation différentielle qui soit bornée dans le secteur considéré. De plus, le terme non homogène est d'ordre au plus $-X^{2}-X u_{0}(X)=o(1)$; on peut en déduire, comme pour $Y_{0}$, que $Y_{1}(X)$ tend vers 0 en l'infini.

On pose maintenant $Z\left(X, \varepsilon^{\prime}\right)=Y_{1}(X)+\varepsilon^{\prime} Z_{1}\left(X, \varepsilon^{\prime}\right)$.

Alors $Z_{1}\left(X, \varepsilon^{\prime}\right)$ est solution de

$$
\begin{aligned}
\varepsilon^{\prime} Z_{1}^{\prime} & =u_{0}\left(\frac{a}{\varepsilon^{\prime}}\right)+\varepsilon^{\prime} h\left(Z, X, \varepsilon^{\prime}\right)-\left(Y_{1}+\varepsilon^{\prime} Z_{1}\right)\left(u_{0}^{2}\left(2+a-\varepsilon^{\prime} X\right)\right)+2 Y_{1} u_{0}^{2} \\
Z_{1}^{\prime} & =-Z_{1}\left(2+a-\varepsilon^{\prime} X\right) u_{0}^{2}+u_{0}\left(\frac{a}{\varepsilon^{\prime 2}}\right)+u_{0}^{2} Y_{1}\left(X-\frac{a}{\varepsilon^{\prime}}\right)+h\left(Z, X, \varepsilon^{\prime}\right),
\end{aligned}
$$

ce qui est une équation différentielle linéaire en $Z_{1}$ (le fait que $Z$ puisse s'écrire en fonction de $Z_{1}$ n'est pas gênant: on sait que cette fonction $Z$ existe, et $h\left(Z, X, \varepsilon^{\prime}\right)$ peut être considéré comme une donnée, comme fonction de $\mathfrak{H})$.

On peut résoudre cette équation:

$$
\begin{aligned}
Z_{1}\left(X, \varepsilon^{\prime}\right)=e^{F\left(X, \varepsilon^{\prime}\right)} \int_{\gamma_{X}} e^{-F\left(t, \varepsilon^{\prime}\right)}\left(u_{0}(t)\right. & \left(\frac{a}{\varepsilon^{\prime 2}}\right) \\
& \left.+u_{0}^{2}(t) Y_{1}(t)\left(t-\frac{a}{\varepsilon^{\prime}}\right)+h\left(Z, t, \varepsilon^{\prime}\right)\right) d t,
\end{aligned}
$$

en rappelant que $F\left(X, \varepsilon^{\prime}\right)=\int^{X}-u_{0}^{2}(t)\left(2+a-\varepsilon^{\prime} t\right) d t$.

Alors, puisque $\left|u_{0}(t)\left(2+a-\varepsilon^{\prime} t\right)\right|>0$ dans $\mathcal{D}$, et que les fonctions $\frac{h\left(Z\left(t, \varepsilon^{\prime}\right), t, \varepsilon^{\prime}\right)}{u_{0}(t)}$ et $Y_{1}(t) u_{0}(t)$ sont bornées, il est clair que $Z_{1}\left(X, \varepsilon^{\prime}\right)$ est une fonction bornée, qui reste dans l'espace $\mathfrak{Y}$. Ce qui démontre le cas $N=1$.

Pour la suite de la démonstration par récurrence, elle devient évidente à partir de l'équation différentielle obtenue pour $Z_{1}$ : on va trouver à chaque fois $Z_{n-1}\left(X, \varepsilon^{\prime}\right)=Y_{n-1}(X)+\varepsilon^{\prime} Z_{n}$, puis

$$
Z_{n}^{\prime}=-Z_{1}\left(2+a-\varepsilon^{\prime} X\right) u_{0}^{2}+h_{n}\left(X, \varepsilon^{\prime}\right),
$$

avec $h_{n}\left(X, \varepsilon^{\prime}\right)=\frac{h_{n-1}\left(X, \varepsilon^{\prime}\right)-h_{n-1}(X, 0)}{\varepsilon^{\prime}}+2 u_{0}^{2}(X) Y_{n-1}(X) . h_{n}$ est une fonction de $\mathfrak{H}$ avec l'hypothèse de récurrence qu'on peut exprimer comme ceci: $h_{n} \in \mathfrak{H}$ et $Z_{n-1} \in \mathfrak{Y}$. Donc, en résolvant l'équation, on voit à nouveau que $Z_{n}\left(X, \varepsilon^{\prime}\right)$ est une fonction de $\mathfrak{Y}$.

Ce qui suffit à démontrer le théorème. 
Éric Matzinger

\section{Estimation de $\alpha^{+}-\alpha^{-}$}

\subsection{Préliminaires}

\subsubsection{Premiers termes du développement de $v(u, \varepsilon)$ et $Y(X, \varepsilon)$}

Le calcul des premiers termes des séries pour $a$ et $v(\mathrm{cf}(1))$ nous donne:

$$
a(\varepsilon)=a_{1} \varepsilon+a_{2} \varepsilon^{2}+\mathcal{O}\left(\varepsilon^{3}\right) \quad v(u, \varepsilon)=v_{0}(u)+\varepsilon v_{1}(u)+\varepsilon^{2} v_{2}(u)+\mathcal{O}\left(\varepsilon^{3}\right)
$$

où

et

$$
a_{1}=-\frac{1}{8} \quad a_{2}=-\frac{3}{32}
$$

$$
\begin{gathered}
v_{0}(u)=\frac{-1}{u+1} \quad v_{1}(u)=-\frac{1}{8} \frac{u^{2}+4 u+7}{(u+1)^{4}} \\
v_{2}(u)=-\frac{1}{32} \frac{3 u^{5}+21 u^{4}+66 u^{3}+126 u^{2}+159 u+121}{(u+1)^{7}}
\end{gathered}
$$

Par récurrence, on démontre que pour $n>0$ les $v_{n}(u)$ sont des fractions rationnelles, dont le numérateur est un polynôme de degré $3 n-1$, et dont le dénominateur est $(u+1)^{3 n+1}$.

Le simple changement de variable $u=-1+\varepsilon^{1 / 3} X$ dans le développement de $v(u, \varepsilon)$ nous donne alors, après simplification:

$$
\begin{aligned}
\varepsilon^{1 / 3} v= & -\frac{1}{X}-\frac{1}{2 X^{4}}-\frac{5}{4 X^{7}}-\varepsilon^{1 / 3}\left(\frac{1}{4 X^{3}}+\frac{9}{8 X^{6}}\right)-\varepsilon^{2 / 3}\left(\frac{1}{8 X^{2}}+\frac{3}{4 X^{5}}\right) \\
& -\varepsilon \frac{3}{8 X^{4}}-\varepsilon^{4 / 3} \frac{3}{16 X^{3}}-\varepsilon^{5 / 3} \frac{3}{32 X^{2}}+\mathcal{O}\left(\frac{1}{|X|^{8}}+|\varepsilon| \frac{1}{|X|^{5}}+|\varepsilon|^{2} \frac{1}{|X|^{2}}\right) .
\end{aligned}
$$

Ensuite, comme $Y(X, \varepsilon)=\varepsilon^{1 / 3} v$ se met sous la forme

$$
Y=Y_{0}+\varepsilon^{1 / 3} Y_{1}+\cdots+Y_{n} \varepsilon^{n / 3}+o\left(\varepsilon^{n / 3}\right),
$$

après identification, on trouve pour les $Y_{n}$ :

$$
\begin{aligned}
& Y_{0}=-\frac{1}{X}-\frac{1}{2 X^{4}}+\mathcal{O}\left(\frac{1}{X^{7}}\right) \\
& Y_{1}=-\frac{1}{4 X^{3}}+\mathcal{O}\left(\frac{1}{X^{6}}\right) \\
& Y_{2}=-\frac{1}{8 X^{2}}+\mathcal{O}\left(\frac{1}{X^{5}}\right) \\
& Y_{3}=\mathcal{O}\left(\frac{1}{X^{4}}\right)
\end{aligned}
$$


et pour $n \geqslant 4$, on a

$$
Y_{n}=\mathcal{O}\left(\frac{1}{X^{2}}\right) \text { ou } Y_{n}=o\left(\frac{1}{X^{2}}\right) .
$$

\subsection{2. Équivalent de $Y_{0}^{+}-Y_{0}^{-}$}

On introduit les fonctions suivantes:

$$
\mathrm{Ai}_{0}=\mathrm{Ai}, \quad \mathrm{Ai}_{1}: x \mapsto \mathrm{Ai}(j x), \quad \mathrm{Ai}_{2}: x \mapsto \mathrm{Ai}\left(j^{2} x\right)
$$

On cherche à estimer la différence de deux des solutions de (2). Pour cela, nous nous intéressons aux deux fonctions suivantes:

$$
X_{1}(z)=-2 \frac{\mathrm{Ai}_{1}^{\prime}(z)}{\mathrm{Ai}_{1}(z)} \quad \text { et } \quad X_{2}(z)=-2 \frac{\mathrm{Ai}_{2}^{\prime}(z)}{\mathrm{Ai}_{2}(z)}
$$

Ces deux fonctions vérifient, à cause de la symétrie par rapport à l'axe réel de l'équation (3), la relation $X_{1}(z)=\overline{X_{2}(\bar{z})}$. Elles correspondent à deux solutions de l'équation (2):

$$
Y_{0}^{+}(X)=X_{2}^{2}(z(X))+z(X) \quad \text { et } \quad Y_{0}^{-}(X)=X_{1}^{2}(z(X))+z(X) .
$$

En particulier, pour $X_{1}(z)$ sur l'axe réel, on a:

$$
\begin{aligned}
X: & =X_{1}(z)=X_{1}\left(Y_{0}^{-}\left(X_{1}(z)\right)-X_{1}^{2}(z)\right)=X_{1}\left(Y_{0}^{-}(X)-X^{2}\right) \\
& =X_{2}(\bar{z})=X_{2}\left(\overline{Y_{0}^{-}(X)-X^{2}}\right)=X_{2}\left(Y_{0}^{+}(X)-X^{2}\right) .
\end{aligned}
$$

On note dans ce cas

$$
z^{+}(X)=Y_{0}^{+}(X)-X^{2} \quad \text { et } \quad z^{-}(X)=Y_{0}^{-}(X)-X^{2}
$$

On cherche un équivalent de $Y_{0}^{+}-Y_{0}^{-}$, et nous allons montrer le résultat suivant:

Lemme 4. - Pour X sur l'axe réel,

$$
\left(Y_{0}^{+}-Y_{0}^{-}\right)(X) \underset{X \rightarrow+\infty}{\sim} \frac{4}{e} X^{2} e^{-\frac{2}{3} X^{3}} i .
$$


Ce qui revient à réussir à calculer exactement un coefficient de Stokes pour une équation différentielle non linéaire; dans le cas général, ce n'est pas possible.

Pour obtenir ce résultat, on utilise le fait que pour $X$ réel et suffisamment grand, $Y_{0}^{ \pm}-X^{2}$ est presque un réel négatif. Dans ce cas $\left|\mathrm{Ai}_{k}\left(Y_{0}^{ \pm}-X^{2}\right)\right|$ est grand pour $k=1$ et $k=2$, exponentiellement petit par contre pour $k=0$, ce qui justifie l'utilisation d'un développement limité pour estimer les deux fonctions $\mathrm{Ai}_{2}(z)=-j^{2} \mathrm{Ai}_{1}(z)-j \mathrm{Ai}_{0}(z)$ et $\mathrm{Ai}_{2}^{\prime}(z)=-j^{2} \mathrm{Ai}_{1}^{\prime}(z)-j \mathrm{Ai}_{0}^{\prime}(z)$. Dans le calcul qui suit, on travaillera en variable $X$, avec aussi les fonctions $z^{ \pm}(X)$ introduites plus haut.

$$
\begin{aligned}
X_{1}\left(Y_{0}^{-}(X)-X^{2}\right) & =X_{2}\left(Y_{0}^{+}(X)-X^{2}\right) \\
-2 \frac{A i_{1}^{\prime}\left(z^{-}\right)}{\mathrm{Ai}_{1}\left(z^{-}\right)} & =-2 \frac{\mathrm{Ai}_{2}^{\prime}\left(z^{+}\right)}{\mathrm{Ai}_{2}\left(z^{+}\right)} \\
-2 \frac{\mathrm{Ai}_{1}^{\prime}\left(z^{-}\right)}{A i_{1}\left(z^{-}\right)} & =-2 \frac{\mathrm{Ai}_{1}^{\prime}\left(z^{+}\right)+j^{2} \mathrm{Ai}_{0}^{\prime}\left(z^{+}\right)}{\mathrm{Ai}_{1}\left(z^{+}\right)+j^{2} \mathrm{Ai}_{0}\left(z^{+}\right)} \\
X_{1}\left(z^{-}\right) & =X_{1}\left(z^{+}\right)\left(1+j^{2} \frac{\mathrm{Ai}_{0}^{\prime}\left(z^{+}\right)}{\mathrm{Ai}_{1}^{\prime}\left(z^{+}\right)}-j^{2} \frac{\mathrm{Ai}_{0}\left(z^{+}\right)}{\mathrm{Ai}_{1}\left(z^{+}\right)}+o(\ldots)\right) \\
X_{1}\left(z^{-}\right)-X_{1}\left(z^{+}\right) & =X_{1}\left(z^{+}\right)\left(j^{2} \frac{\mathrm{Ai}_{0}^{\prime}\left(z^{+}\right)}{\mathrm{Ai}_{1}^{\prime}\left(z^{+}\right)}-j^{2} \frac{\mathrm{Ai}_{0}\left(z^{+}\right)}{\mathrm{Ai}_{1}\left(z^{+}\right)}+o(\ldots)\right)
\end{aligned}
$$

Il va être possible de calculer un équivalent de cette différence, ce qui nous mènera à un équivalent pour $Y_{0}^{+}-Y_{0}^{-}$. En effet, avec une formule de Taylor

$$
X_{1}\left(z^{-}\right)-X_{1}\left(z^{+}\right) \sim\left(z^{-}-z^{+}\right) X_{1}^{\prime}\left(z^{-}\right)=\left(Y_{0}^{--}-Y_{0}^{+}\right) X_{1}^{\prime}\left(z^{-}\right)
$$

sachant que d'après l'équation différentielle pour $X_{1}$, on peut écrire sa dérivée

$$
\begin{aligned}
X_{1}^{\prime}\left(z^{-}\right) & =\frac{X_{1}^{2}\left(z^{-}\right)+z^{-}}{2} \\
X_{1}^{\prime}\left(z^{-}(X)\right) & =\frac{X^{2}+\left(Y_{0}^{-}(X)-X^{2}\right)}{2} \sim-\frac{1}{2 X} .
\end{aligned}
$$

Nous allons utiliser les équivalents déjà vus précédemment $(\mathrm{cf}(4))$ pour estimer les deux fractions $\frac{A i_{0}^{\prime}\left(z^{+}\right)}{A i_{1}^{\prime}\left(z^{+}\right)}$et $\frac{A i_{0}\left(z^{+}\right)}{A i_{1}\left(z^{+}\right)}$, puisque $z^{ \pm}$est proche du demi-axe réel négatif quand $X \rightarrow \infty$ :

$$
\xi=\frac{2}{3}\left(\mu\left(X^{2}-Y_{0}(X)\right)\right)^{3 / 2}=\frac{2}{3}\left(2^{-2 / 3}\right)^{3 / 2}\left(X^{2}+\frac{1}{X}+\mathcal{O}\left(\frac{1}{X^{4}}\right)\right)^{3 / 2}
$$


Étude des solutions surstables de l'équation de Van der Pol

$$
\begin{gathered}
=\frac{1}{3}\left(X^{3}+\frac{3}{2}+o(1)\right) \\
\begin{aligned}
\xi_{2}=\frac{2}{3}\left(j \mu\left(X^{2}-Y_{0}(X)\right)\right)^{3 / 2} & =e^{i \pi} \xi=-\xi \\
\frac{\mathrm{Ai}_{0}^{\prime}\left(z^{+}\right)}{\mathrm{Ai}_{1}^{\prime}\left(z^{+}\right)}-\frac{\mathrm{Ai}_{0}\left(z^{+}\right)}{\mathrm{Ai}_{1}\left(z^{+}\right)} & \sim \frac{-e^{-\xi} z^{1 / 4}}{1} \times \frac{1}{-j(j z)^{1 / 4} e^{\xi}}-\frac{e^{-\xi}}{z^{1 / 4}} \times \frac{(j z)^{1 / 4}}{e^{\xi}} \\
& \sim e^{-5 i \pi / 6} e^{-2 \xi}+e^{-5 i \pi / 6} e^{-2 \xi} \\
& \sim 2 e^{-5 i \pi / 6} e^{-2 \xi}
\end{aligned}
\end{gathered}
$$

En rappelant que

$$
\left(Y_{0}^{+}-Y_{0}^{-}\right) X_{1}^{\prime}\left(z^{-}\right) \sim-X_{1}\left(z^{+}\right) j^{2}\left(\frac{\mathrm{Ai}_{0}^{\prime}\left(z^{+}\right)}{\mathrm{Ai}_{1}^{\prime}\left(z^{+}\right)}-\frac{\mathrm{Ai}_{0}\left(z^{+}\right)}{\mathrm{Ai}_{1}\left(z^{+}\right)}\right)
$$

on trouve comme équivalent pour la différence le résultat annoncé au lemme 4:

$$
\left(Y_{0}^{+}-Y_{0}^{-}\right)(X) \sim \frac{4}{e} X^{2} e^{-\frac{2}{3} X^{3}} i \quad \text { quand } X \rightarrow \infty \text { dans R. }
$$

\section{2. Établissement de l'équation vérifiée par $\alpha^{+}-\alpha^{-}$}

On rappelle l'équation (E), vérifiée pour $(v, \alpha)=\left(v^{+}, \alpha^{+}\right)$et $\left(v^{-}, \alpha^{-}\right)$.

$$
\text { (E) } \varepsilon \frac{d v}{d u}=\left(1-u^{2}\right)+\frac{\alpha-u}{v} \text {. }
$$

Le but des paragraphes à venir va être d'obtenir une estimation de $\alpha^{+}-$ $\alpha^{-}$. Pour cela, on commence par chercher une équation différentielle en $v^{+}-v^{-}$, où le terme à calculer sera présent. On pose alors

$$
\begin{array}{ll}
b=\alpha^{+}-\alpha^{-} & c=\alpha^{+}+\alpha^{-} \\
d=v^{+}-v^{-} & e=v^{+}+v^{-}
\end{array}
$$

Quand on effectue la substitution dans l'équation (E), on obtient

$$
\begin{aligned}
& \varepsilon d^{\prime}=\left(1-u^{2}\right)+\frac{\alpha^{+}-u}{v^{+}}-\left(1-u^{2}\right)-\frac{\alpha^{-}-u}{v^{-}} \\
&=\frac{u d+\alpha^{+} v^{-}-\alpha^{-} v^{+}}{v^{+} v^{-}} \\
&=\frac{u}{v^{+} v^{-}} d+\frac{\frac{b+c}{2} \cdot \frac{e-d}{2}+\frac{b-c}{2} \cdot \frac{e+d}{2}}{v^{+} v^{-}} \\
&-733-
\end{aligned}
$$




$$
\begin{aligned}
& =\frac{u}{v^{+} v^{-}} d+\frac{e}{2 v^{+} v^{-}} b-\frac{c}{2 v^{+} v^{-}} d \\
& =\frac{u-c / 2}{v^{+} v^{-}} d+\frac{v^{+}+v^{-}}{2 v^{+} v^{-}} b
\end{aligned}
$$

En remplaçant $c=\alpha^{+}+\alpha^{-}=1+a^{+}+1+a^{-}$, on obtient en définitive l'équation voulue.

LEMme 5. - On pose $d(u)=v^{+}(u)-v^{-}(u)$ et $b=\alpha^{+}-\alpha^{-}$. Alors l'équation différentielle suivante est vérifiée:

$$
\varepsilon d^{\prime}(u)=f(u) d(u)+g(u) b
$$

avec

$$
f(u)=\frac{u-1-a}{v^{+} v^{-}} \quad \text { et } \quad g(u)=\frac{v^{+}+v^{-}}{2 v^{+} v^{-}}
$$

sachant que

$$
a=\frac{a^{+}+a^{-}}{2}=-\frac{1}{8} \varepsilon+\mathcal{O}\left(\varepsilon^{2}\right) .
$$

(toutes les fonctions ici dépendent aussi de $\varepsilon$, cette dépendance n'est pas rappelée).

On intègre ensuite cette équation différentielle avec la méthode de la variation de la constante, entre $+\infty$ et $x\left(\operatorname{car} \lim _{+\infty} d=0\right)$ :

$$
d(x)=\frac{b}{\varepsilon} e^{F(x) / \varepsilon} \int_{+\infty}^{x} e^{-F(u) / \varepsilon} g(u) d u,
$$

où

$$
F(t)=\int_{1}^{t} f(u) d u
$$

Le choix de $x=x_{l}=-1+X_{l} \varepsilon^{1 / 3}$ permet de trouver que

$$
b=\varepsilon d\left(x_{l}\right) e^{-F\left(x_{l}\right) / \varepsilon} \frac{1}{\int_{+\infty}^{x_{l}} e^{-F(u) / \varepsilon} g(u) d u} .
$$

\subsection{Calcul de l'équivalent pour $b$}

On peut trouver une estimation, quand $\varepsilon \rightarrow 0$, de chacun des facteurs dans le produit de l'égalité (12); le but final étant d'en obtenir une pour $b$. 
Tout d'abord, on a vu que jusqu'au point $u=x_{l}$, on pouvait affirmer que la fonction $v^{+}(u)=\varepsilon^{-1 / 3} Y^{+}\left(\varepsilon^{-1 / 3}(1+u)\right)$ était équivalente à $\varepsilon^{-1 / 3} Y_{0}^{+}\left(\varepsilon^{-1 / 3}(1+u)\right)$ quand $\varepsilon \rightarrow 0$. Ce qui nous mène à

$$
d\left(x_{l}\right)=v^{+}\left(x_{l}\right)-v^{-}\left(x_{l}\right) \underset{\varepsilon \rightarrow 0}{\sim} \varepsilon^{-1 / 3}\left(Y_{0}^{+}\left(X_{l}\right)-Y_{0}^{-}\left(X_{l}\right)\right) .
$$

Ensuite, l'intégrale présente dans (12) est susceptible d'être estimée avec la méthode du point col. On constate d'abord que

$$
F^{\prime}(t)=f(t)=0 \Longrightarrow t=1+a=\alpha .
$$

Or on vérifie sans difficulté que $F(\alpha)=\mathcal{O}\left(\varepsilon^{2}\right)$. D'où

$$
\frac{F(t)}{\varepsilon}=\mathcal{O}(\varepsilon)+\frac{(t-\alpha)^{2}}{2 \varepsilon} f^{\prime}(\alpha)+\frac{\mathcal{O}(t-\alpha)^{3}}{\varepsilon} .
$$

Sachant que $v^{ \pm}(\alpha)=-\frac{1}{2}+\mathcal{O}(\varepsilon)$, on trouve

$$
\begin{aligned}
f^{\prime}(\alpha) & =\frac{1}{v^{+}(\alpha) v^{-}(\alpha)}-\left(v^{+} v^{-}\right)^{\prime}(\alpha) \frac{\alpha-1-a}{\left(v^{+}(\alpha) v^{-}(\alpha)\right)^{2}} \\
& =\left(\frac{1}{(-1 / 2)^{2}}+\mathcal{O}(\varepsilon)\right)+0 \\
& =4+\mathcal{O}(\varepsilon),
\end{aligned}
$$

donc

$$
\frac{F(t)}{\varepsilon}=\frac{2(t-\alpha)^{2}}{\varepsilon}+\frac{\mathcal{O}\left(|t-\alpha|^{3}+|\varepsilon| \cdot|t-\alpha|^{2}\right)}{\varepsilon}
$$

Par ailleurs

$$
g(\alpha) \sim-2 .
$$

La contribution principale dans l'intégrale vient du voisinage de $\alpha$, on va donc écrire, après le changement de variable $x=(t-\alpha) \sqrt{2 / \varepsilon}$,

$$
\begin{aligned}
-\int_{x_{l}}^{+\infty} g(t) e^{-F(t) / \varepsilon} d t & \sim-\sqrt{\frac{\varepsilon}{2}} \int_{\left(x_{l}-\alpha\right) \sqrt{2 / \varepsilon}}^{+\infty} g(\alpha+x \sqrt{\varepsilon / 2}) e^{-F(\alpha+x \sqrt{\varepsilon / 2}) / \varepsilon} d x \\
& \sim-\sqrt{\frac{\varepsilon}{2}} g(\alpha) \int_{(-2) \sqrt{2 / \varepsilon}}^{+\infty} e^{-x^{2}+\sqrt{\varepsilon} \cdot \mathcal{O}\left(|x|^{3}+\sqrt{|\varepsilon| \cdot|x|^{2}}\right)} d x \\
& \sim 2 \sqrt{\frac{\varepsilon}{2}} \int_{-\infty}^{+\infty} e^{-x^{2}} d x .
\end{aligned}
$$


Un équivalent de l'intégrale est donc:

$$
-\int_{x_{l}}^{+\infty} g(t) e^{-F(t) / \varepsilon} d t \sim \sqrt{2 \pi \varepsilon}
$$

Il reste à trouver une estimation pour $F\left(x_{l}\right)$. Pour calculer $F\left(x_{l}\right)$, on peut couper l'intervalle $\left[x_{l}, 1\right]$ en deux parties, en un point $(-1+\lambda)$, où $\lambda$ est un réel fixé à choisir entre 0 et 2 . À gauche, on pourra alors raisonnablement estimer $v(u)$ par l'expression $\varepsilon^{-1 / 3}\left(Y_{0}(X)+\varepsilon^{1 / 3} Y_{1}(X)+\varepsilon^{2 / 3} Y_{2}(X)+\mathcal{O}(\varepsilon)\right)$ d'après le corollaire 3 , et à droite par $v_{0}+\varepsilon v_{1}+\mathcal{O}\left(\varepsilon^{2}\right)$.

$$
\frac{1}{\varepsilon} F\left(x_{l}\right)=\underbrace{\frac{1}{\varepsilon} \int_{1}^{-1+\lambda} \frac{u-1-a}{v^{+}(u) v^{-}(u)} d u}_{(A)}+\underbrace{\frac{1}{\varepsilon} \int_{-1+\lambda}^{x_{l}} \frac{u-1-a}{v^{+}(u) v^{-}(u)} d u}_{(B)}
$$

Les calculs des premières fonctions $v_{n}$ et les approximations pour les $Y_{n}$ (cf. (10)) données dans les préliminaires permettent de donner une estimation des deux intégrales.

Pour l'expression $(A)$, on part de

$$
\frac{1}{v^{+}(u) v^{-}(u)}=(u+1)^{2}\left(1-\frac{\varepsilon}{4} \frac{u^{2}+4 u+7}{(u+1)^{3}}\right)+\mathcal{O}\left(\varepsilon^{2}\right)
$$

puisque $v^{+}$et $v^{-}$ont le même développement asymptotique, d'où

$$
\begin{aligned}
(A) & =\frac{1}{\varepsilon} \int_{1}^{-1+\lambda} \frac{u-1-a}{v^{+}(u) v^{-}(u)} d u \\
& =\frac{1}{\varepsilon} \int_{1}^{-1+\lambda}(u-1-a)(u+1)^{2} d u \\
& =-\frac{1}{4} \int_{1}^{-1+\lambda}(u-1-a) \frac{u^{2}+4 u+7}{u+1} d u+\mathcal{O}(\varepsilon) .
\end{aligned}
$$

On rappelle que $a=-\varepsilon / 8+\mathcal{O}\left(\varepsilon^{2}\right)$. Le calcul des intégrales nous donne alors

$$
(A)=\frac{1}{\varepsilon}\left(\frac{4}{3}-\frac{2 \lambda^{3}}{3}+\frac{\lambda^{4}}{4}\right)+\left(\frac{1}{3}-\frac{\lambda^{3}}{24}+2 \ln \lambda-2 \ln (2)\right)+\mathcal{O}(\varepsilon) .
$$

Pour $(B)$, le calcul se révèle plus complexe. On sait que chacune des deux fonctions $v^{+}$et $v^{-}$se prolonge à l'aide d'une fonction du type $Y_{\varepsilon}$ du théorème 2 :

$$
v^{ \pm}(u, \varepsilon)=\varepsilon^{-1 / 3} Y_{\varepsilon}^{ \pm}\left(\frac{u}{\varepsilon^{\prime}}\right)
$$


avec $X=u / \varepsilon^{\prime}$, en utilisant le corollaire 3 ,

$v^{ \pm}(u, \varepsilon)$

$$
=\varepsilon^{-1 / 3}\left(Y_{0}^{ \pm}(X)+\varepsilon^{1 / 3} Y_{1}^{ \pm}(X)+\varepsilon^{2 / 3} Y_{2}^{ \pm}(X)+\varepsilon Y_{3}^{ \pm}(X)+\frac{\varepsilon^{4 / 3}}{X} \mathcal{O}_{\varepsilon}(1)\right)
$$

où le $\mathcal{O}_{\varepsilon}(1)$ est borné quand $\varepsilon \rightarrow 0$ et au moins borné quand $X \rightarrow \infty$.

Comme $v^{+}$et $v^{-}$ont le même développement asymptotique pour tout $u>\rho$ dans un certain secteur autour de l'axe réel, il est clair que le développement en $1 / X$ des $Y_{n}$ donné plus haut est valable pour $Y_{n}^{+}$comme pour $Y_{n}^{-}$. Ces deux fonctions sont donc exponentiellement proches en $1 / X$ quand $X$ devient grand. On peut donc écrire,

$$
\begin{aligned}
(B) & =\frac{1}{\varepsilon} \int_{-1+\lambda}^{x_{l}} \frac{u-1-a}{v^{+}(u) v^{-}(u)} d u \\
& =\int_{\lambda \varepsilon^{-1 / 3}}^{X_{l}} \frac{-2+\varepsilon^{1 / 3} X-a}{Y_{0}^{+}(X) Y_{0}^{-}(X)}\left(1-\varepsilon^{1 / 3} Q_{1}(X)+\varepsilon^{2 / 3} Q_{2}(X)+\mathrm{R}(X, \varepsilon)\right) d X,
\end{aligned}
$$

en notant

$$
Q_{1}=\frac{Y_{1}^{+}}{Y_{0}^{+}}+\frac{Y_{1}^{-}}{Y_{0}^{-}}, Q_{2}=\frac{Y_{1}^{+^{2}}}{Y_{0}^{+^{2}}}+\frac{Y_{1}^{+} Y_{1}^{-}}{Y_{0}^{+} Y_{0}^{-}}+\frac{Y_{1}^{-2}}{Y_{0}^{-2}}-\frac{Y_{2}^{+}}{Y_{0}^{+}}-\frac{Y_{2}^{-}}{Y_{0}^{-}}
$$

le reste $\mathrm{R}(X, \varepsilon)$ se trouve être de la forme $\left(\frac{\varepsilon}{X}+\varepsilon^{4 / 3}\right) \mathcal{O}_{\varepsilon}(1)$, puisqu'il correspond à des termes $\varepsilon \frac{Y_{k}^{ \pm}}{Y_{0}^{ \pm}}(1 \leqslant k \leqslant 3)$ et $\frac{\varepsilon^{4 / 3}}{X} \frac{1}{Y_{0}^{ \pm}}$respectivement équivalents quand $X$ devient grand à $\frac{\varepsilon}{X}$ et $\varepsilon^{4 / 3}$.

$$
\begin{aligned}
(B)= & \int_{\lambda \varepsilon^{-1 / 3}}^{X_{l}} \frac{-2+\varepsilon^{1 / 3} X-a}{Y_{0}^{+}(X) Y_{0}^{-}(X)} d X-\varepsilon^{1 / 3} \int_{\lambda \varepsilon^{-1 / 3}}^{X_{l}} \frac{-2+\varepsilon^{1 / 3} X-a}{Y_{0}^{+} Y_{0}^{-}} Q_{1} d X \\
& +\varepsilon^{2 / 3} \int_{\lambda \varepsilon^{-1 / 3}}^{X_{l}} \frac{-2+\varepsilon^{1 / 3} X-a}{Y_{0}^{+} Y_{0}^{-}} Q_{2} d X+\int_{\lambda \varepsilon^{-1 / 3}}^{X_{l}} \frac{\left(-2+\varepsilon^{1 / 3} X-a\right) \mathrm{R}(X, \varepsilon)}{Y_{0}^{+} Y_{0}^{-}} d X
\end{aligned}
$$

On appellera les quatre intégrales précédentes, dans l'ordre, $(I),(I I),(I I I)$ et $(I V)$.

Pour $(I)$, regardons d'abord $\int_{\lambda \varepsilon^{-1 / 3}}^{X_{l}} \frac{-2}{Y_{0}^{+} Y_{0}^{-}} d X$. D'après l'équation différentielle (2) vérifiée par $Y_{0}$, on a

$$
\begin{aligned}
\left(Y_{0}^{+}-Y_{0}^{-}\right)^{\prime}(X) & =\frac{-2}{Y_{0}^{+}(X) Y_{0}^{-}(X)}\left(Y_{0}^{+}-Y_{0}^{-}\right)(X) \\
\Longrightarrow\left(Y_{0}^{+}-Y_{0}^{-}\right)(X) & =\left(Y_{0}^{+}-Y_{0}^{-}\right)(0) e^{\int_{0}^{X} \frac{-2}{Y_{0}^{+} Y_{0}^{-}} d X} \quad \text { (en intégrant l'équation). }
\end{aligned}
$$




\section{Éric Matzinger}

Or, quand $X \longrightarrow \infty$, on a vu avec (11) que

$$
\left(Y_{0}^{+}-Y_{0}^{-}\right)(X) \sim \frac{4}{e} i X^{2} e^{-\frac{2}{3} X^{3}},
$$

donc

$$
\begin{gathered}
\int_{\lambda \varepsilon^{-1 / 3}}^{X_{l}} \frac{-2}{Y_{0}^{+} Y_{0}^{-}} d X=\ln \left(\frac{\left(Y_{0}^{+}-Y_{0}^{-}\right)\left(X_{l}\right)}{i}\right)-\ln \left(\frac{\left(Y_{0}^{+}-Y_{0}^{-}\right)\left(\lambda \varepsilon^{-1 / 3}\right)}{i}\right) \\
=\ln \left(-i\left(Y_{0}^{+}-Y_{0}^{-}\right)\left(X_{l}\right)\right)+1-2 \ln 2+\frac{2 \lambda^{3}}{3 \varepsilon}-2 \ln \left(\lambda \varepsilon^{-1 / 3}\right)+o(1) \\
=\ln \left(-i\left(Y_{0}^{+}-Y_{0}^{-}\right)\left(X_{l}\right)\right)+\frac{2 \lambda^{3}}{3} \frac{1}{\varepsilon}+\frac{2}{3} \ln \varepsilon-2 \ln 2 \lambda+1+o(1) .
\end{gathered}
$$

Pour tous les termes à venir (y compris pour les autres intégrales), l'intégration au voisinage de $X_{l}$ et de tout point fini ne donne au final que du $o(\varepsilon)$, et il n'y a donc que ce qui se passe quand $X \rightarrow \infty$ qui nous intéresse. Autrement dit, la différence entre $Y_{n}^{ \pm}$et son développement en $1 / X$ est négligée. Ainsi, pour $\int_{\lambda \varepsilon^{-1 / 3}}^{X_{l}} \frac{\varepsilon^{1 / 3} X}{Y_{0}^{+} Y_{0}^{-}} d X$, on utilise le développement asymptotique de $Y_{0}$ :

$$
\begin{aligned}
Y_{0}=-\frac{1}{X}-\frac{1}{2 X^{4}}+\mathcal{O}\left(\frac{1}{X^{7}}\right) & \Longrightarrow \frac{1}{Y_{0}^{2}}=X^{2}-\frac{1}{X}+\mathcal{O}\left(\frac{1}{X^{4}}\right) \\
\int_{\lambda \varepsilon^{-1 / 3}}^{X_{1}} \frac{\varepsilon^{1 / 3} X}{Y_{0}^{+} Y_{0}^{-}} d X & =\varepsilon^{1 / 3} \int_{\lambda \varepsilon^{-1 / 3}}\left(X^{3}-1\right) d X+\mathcal{O}\left(\varepsilon^{1 / 3}\right) \\
& =-\varepsilon^{1 / 3} \frac{\lambda^{4}}{4 \varepsilon^{4 / 3}}+\varepsilon^{1 / 3} \lambda \varepsilon^{-1 / 3}+\mathcal{O}\left(\varepsilon^{1 / 3}\right) \\
& =-\frac{1}{\varepsilon} \frac{\lambda^{4}}{4}+\lambda+\mathcal{O}\left(\varepsilon^{1 / 3}\right)
\end{aligned}
$$

Et pour la dernière partie de $(I)$,

$$
\begin{aligned}
\int_{\lambda \varepsilon^{-1 / 3}}^{X_{l}} \frac{a}{Y_{0}^{+} Y_{0}^{-}} d X & =-\frac{\varepsilon}{16} \int_{\lambda \varepsilon^{-1 / 3}}^{X_{l}} \frac{-2}{Y_{0}^{+} Y_{0}^{-}} d X(1+\mathcal{O}(\varepsilon)) \\
& =-\frac{\lambda^{3}}{24}+o(1) .
\end{aligned}
$$

D'où finalement

$$
\begin{aligned}
(I)=\frac{1}{\varepsilon}\left(\frac{2 \lambda^{3}}{3}-\frac{\lambda^{4}}{4}\right)+\frac{2}{3} \ln \varepsilon-2 \ln \lambda & -2 \ln 2+1+\lambda \\
& -\frac{\lambda^{3}}{24}+\ln \left(\frac{\left(Y_{0}^{+}-Y_{0}^{-}\right)\left(X_{l}\right)}{i}\right)+o(1) .
\end{aligned}
$$


Étude des solutions surstables de l'équation de Van der Pol

On peut procéder de manière tout a fait identique pour $(I I)$

$$
(I I)=\varepsilon^{1 / 3} \int_{\lambda \varepsilon^{-1 / 3}}^{X_{l}} \frac{-2+\varepsilon^{1 / 3} X-a}{Y_{0}^{+} Y_{0}^{-}} Q_{1}(X) d X
$$

en utilisant la série asymtotique de $Y_{1}$ dans $Q_{1}$ et ne négligeant ce qui est en facteur de $a=\mathcal{O}(\varepsilon)$.

$$
\begin{gathered}
Y_{1}(X)=-\frac{1}{4 X^{3}}+\mathcal{O}\left(X^{-6}\right) \Longrightarrow-\frac{2 Y_{1}}{Y_{0}^{3}}=-\frac{1}{2}+o(1) \\
(I I)=\varepsilon^{1 / 3} \int^{\lambda \varepsilon^{-1 / 3}} \frac{-2+\varepsilon^{1 / 3} X}{2} d X+o(1)=\frac{\lambda^{2}}{4}-\lambda+o(1)
\end{gathered}
$$

Et de même pour $(I I I)$.

$$
\begin{gathered}
(I I I)=\varepsilon^{2 / 3} \int_{\lambda \varepsilon^{-1 / 3}}^{X_{l}} \frac{\left(-2+\varepsilon^{1 / 3} X\right)}{Y_{0}^{2}}\left(\frac{Y_{1}^{2}}{Y_{0}^{2}}-2 \frac{Y_{2}}{Y_{0}}\right) d X \\
Y_{2}(X)=-\frac{1}{8 X^{2}}+\mathcal{O}\left(\frac{1}{X^{5}}\right) \Longrightarrow-\frac{2 Y_{2}}{Y_{0}^{3}}=-\frac{X}{4}+\mathcal{O}\left(\frac{1}{X^{2}}\right) ; \operatorname{et} \frac{Y_{1}^{2}}{Y_{0}^{4}}=\mathcal{O}\left(\frac{1}{X^{2}}\right) \\
(I I I)=\varepsilon^{2 / 3} \int^{\lambda \varepsilon^{-1 / 3}}\left(-2+\varepsilon^{1 / 3} X\right)\left(-\frac{X}{4}\right)+o(1)=\frac{\lambda^{3}}{12}-\frac{\lambda^{2}}{4}+o(1)
\end{gathered}
$$

Pour la dernière intégrale,

$$
\begin{aligned}
(I V) & \leqslant \varepsilon \int_{\lambda \varepsilon^{-1 / 3}} \frac{-2+\varepsilon^{1 / 3} X}{Y_{0}^{2}}\left(\frac{1}{X}+\varepsilon^{1 / 3}\right) \mathcal{O}_{\varepsilon}(1) d X \\
& \leqslant \varepsilon \int_{\lambda \varepsilon^{-1 / 3}}\left(\mathcal{O}_{\varepsilon}(X)+\varepsilon^{1 / 3} \mathcal{O}_{\varepsilon}\left(X^{2}\right)\right) d X \\
& \leqslant \varepsilon \mathcal{O}\left(\varepsilon^{-2 / 3}\right) \\
& =o(1)
\end{aligned}
$$

En fin de compte, quand on additionne $(A)+(I)+(I I)+(I I I)+(I V)$, on obtient

$$
\frac{1}{\varepsilon} F\left(x_{l}\right)=\frac{1}{\varepsilon} \frac{4}{3}+\frac{2}{3} \ln \varepsilon+\frac{4}{3}-4 \ln 2+\ln \left(\frac{\left(Y_{0}^{+}-Y_{0}^{-}\right)\left(X_{l}\right)}{i}\right)+o(1)
$$

Donc

$$
e^{-F\left(x_{l}\right) / \varepsilon} \sim e^{-4 / 3 \varepsilon} \varepsilon^{-2 / 3} \frac{16}{e^{4 / 3}} \frac{i}{\left(Y_{0}^{+}-Y_{0}^{-}\right)\left(X_{l}\right)}
$$


Remarque . - On peut signaler que le calcul de $(A)$ ci-dessus n'est pas indispensable et qu'il suffit de poser $\lambda=0$ dans $(B)$ pour obtenir le bon résultat. Le calcul complet de $(A)$ a cependant été conservé parce qu'a priori, il est indispensable, qu'il peut l'être dans d'autres exemples et parce qu'il permet de détecter d'éventuelles erreurs. Dans l'autre sens, remplacer $\lambda$ par $X_{l} \varepsilon^{1 / 3}$ dans $(A)$ ne suffit pas pour obtenir le résultat complet, mais donne déjà les termes $e^{-4 / 3 \varepsilon} \varepsilon^{-2 / 3}$.

On peut en revanche choisir effectivement n'importe quel $\lambda$ fixé dans ]0,2[, ou même un $\lambda$ variant très peu avec $\varepsilon$ comme par exemple $\varepsilon^{1 / 20}$, on aboutira au bon résultat, avec quelques complications dans le second cas.

En combinant les résultats intermédiaires (13), (14) et (15), cela nous donne finalement:

$$
\begin{aligned}
b & \sim i \varepsilon e^{-4 / 3 \varepsilon} \varepsilon^{-2 / 3} \frac{16}{e^{4 / 3}} \frac{\varepsilon^{-1 / 3}\left(Y_{0}^{+}-Y_{0}^{-}\right)\left(X_{l}\right)}{\left(Y_{0}^{+}-Y_{0}^{-}\right)\left(X_{l}\right)} \frac{1}{\sqrt{2 \pi \varepsilon}} \\
b & \sim i \frac{e^{-\frac{4}{3 \varepsilon}}}{\sqrt{\varepsilon}} \frac{8 \sqrt{2}}{\sqrt{\pi} e^{4 / 3}}
\end{aligned}
$$

Soit le

THÉORÈME 6

$$
\alpha^{+}-\alpha^{-} \sim i \frac{e^{-\frac{4}{3 \varepsilon}}}{\sqrt{\varepsilon}} \frac{8 \sqrt{2}}{\sqrt{\pi} e^{4 / 3}}
$$

On retrouve le résultat de [3] de la proximité exponentielle des deux $\alpha$. Et on confirme que la borne trouvée: $\exp (R(-1+\rho) / \varepsilon)$ était bien optimale.

\section{Conséquence sur les coefficients de la série $\hat{\mathbf{a}}(\varepsilon)$}

D'après [3], la fonction $a(\varepsilon)$ est holomorphe dans le domaine

$$
\mathcal{M}=\{\varepsilon / \arg (\varepsilon) \in] \frac{-5 \pi}{2}+\delta, \frac{\pi}{2}-\delta\left[, 0<|\varepsilon| \leqslant\left|\varepsilon_{1}\right|\right\} .
$$

Pour $\varepsilon_{0}$ assez petit $\left(\left|\varepsilon_{0}\right|<\left|\varepsilon_{1}\right|\right)$, le chemin $\gamma$, défini par un arc de cercle de rayon $\left|\varepsilon_{0}\right|$ et deux segments proches de $\left[0, \varepsilon_{0}\right]$ (voir figure 4 ) est inclu dans $\mathcal{M}$, et est rétractile en un point dans ce domaine..

Donc pour $\varepsilon \in \mathcal{M}$, on a

$$
a(\varepsilon)=\frac{1}{2 i \pi} \int_{\gamma} \frac{a(u)}{u-\varepsilon} d u
$$


Étude des solutions surstables de l'équation de Van der Pol

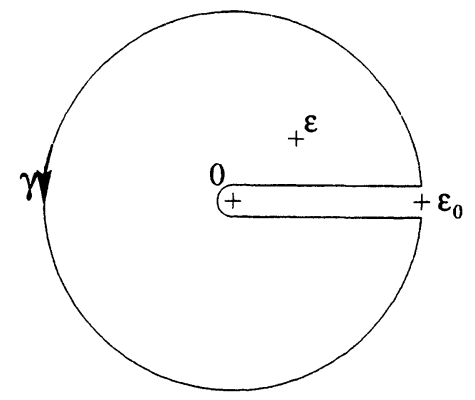

Fig. 4 Chemin d'intégration $\gamma$.

$$
=\frac{1}{2 i \pi} \int_{|u|=\rho} \frac{a(u)}{u-\varepsilon} d u+\frac{1}{2 i \pi} \int_{0}^{\varepsilon_{0}}\left(\frac{a\left(u e^{-2 i \pi}\right)-a(u)}{u-\varepsilon}\right) d u
$$

puis en développant en série de $\varepsilon$ et en identifiant les coefficients obtenus

$$
a_{n}=\frac{1}{2 i \pi} \int_{|u|=\rho} a(u) u^{-n-1} d u+\frac{1}{2 i \pi} \int_{0}^{\varepsilon_{0}}\left(a\left(u e^{-2 i \pi}\right)-a(u)\right) u^{-n-1} d u
$$

comme le premier terme n'est que de l'ordre de $\rho^{-n}$

$$
a_{n} \sim \frac{1}{2 i \pi} \int_{0}^{\varepsilon_{0}}\left(a^{-}(u)-a^{+}(u)\right) u^{-n-1} d u
$$

et que dans cette intégrale, la contribution principale vient du voisinage de 0 , le théorème 6 mène à

$$
a_{n} \sim-\frac{1}{2 i \pi} i \frac{8 \sqrt{2}}{e^{4 / 3} \sqrt{\pi}} \int_{0}^{\varepsilon_{0}} \frac{e^{-4 / 3 u}}{\sqrt{u}} u^{-n-1} d u .
$$

On effectue alors le changement de variable $t=\frac{4}{3 u}, d u=-\frac{4}{3} \frac{d t}{t^{2}}$

$$
a_{n} \sim \frac{1}{2 \pi} \frac{8 \sqrt{2}}{e^{4 / 3} \sqrt{\pi}} \int_{+\infty}^{4 / 3 \varepsilon_{0}} e^{-t} t^{n-1} \sqrt{t} d t \times \sqrt{\frac{3}{4}}\left(\frac{3}{4}\right)^{n} .
$$

Pour $n$ assez grand, $\int_{4 / 3 \varepsilon_{0}}^{0} e^{-t} t^{n-1 / 2} d t$ est négligeable face à $\int_{+\infty}^{4 / 3 \varepsilon_{0}} e^{-t} t^{n-1 / 2} d t$.

Donc

$$
a_{n} \sim \frac{-1}{2 \pi} \frac{8 \sqrt{2}}{e^{4 / 3} \sqrt{\pi}} \sqrt{\frac{3}{4}}\left(\frac{3}{4}\right)^{n} \Gamma\left(n+\frac{1}{2}\right)
$$

Or

$$
\Gamma\left(n+\frac{1}{2}\right) \sim \sqrt{2 \pi n}\left(\frac{n-1 / 2}{e}\right)^{n-1 / 2} \sim \sqrt{2 \pi}\left(\frac{n}{e}\right)^{n}
$$


Donc

$$
a_{n} \sim \frac{-1}{2 \pi} \frac{8 \sqrt{2}}{e^{4 / 3} \sqrt{\pi}} \sqrt{\frac{3}{4}}\left(\frac{3}{4}\right)^{n} \sqrt{2 \pi}\left(\frac{n}{e}\right)^{n}
$$

THÉORÈME 7. - On a un équivalent exact pour les coefficients des $\alpha$ «à canards»:

$$
a_{n} \sim \frac{-4 \sqrt{3}}{\pi e^{4 / 3}}\left(\frac{3 n}{4 e}\right)^{n}
$$

On rappelle que tous les $\alpha$ donnant des solutions «canards» sont exponentiellement proches en $\varepsilon$. Ils ont donc tous le même développement asymptotique. Par ailleurs, le résultat obtenu (à $\varepsilon$ donné) en sommant la série «au plus petit terme» donne aussi une approximation de $\alpha$ exponentiellement bonne, et est donc une valeur à canard [3].

\section{Résultats numériques}

On peut facilement implémenter le calcul de la suite des $a_{n}$ en utilisant les formules de récurrence (1). En particulier, en calculant les 155 premiers termes (calcul effectué par Franck Michel et amicalement transmis) et en regardant le résultat de la multiplication $b_{n}=a_{n} \times(4 e / 3 n)^{n}$, on obtient:

\begin{tabular}{|l|l||l|l||l|l|}
\hline$b_{135}$ & $-0,5417512651$ & $b_{136}$ & $-0,5418690317$ & $b_{137}$ & $-0,5419854885$ \\
\hline$b_{138}$ & $-0,5421006603$ & $b_{139}$ & $-0,5422145711$ & $b_{140}$ & $-0,5423272443$ \\
\hline$b_{141}$ & $-0,5424387024$ & $b_{142}$ & $-0,5425489682$ & $b_{143}$ & $-0,5426580621$ \\
\hline$b_{144}$ & $-0,5427660064$ & $b_{145}$ & $-0,5428728208$ & $b_{146}$ & $-0,5429785257$ \\
\hline$b_{147}$ & $-0,5430831405$ & $b_{148}$ & $-0,5431866841$ & $b_{149}$ & $-0,5432891757$ \\
\hline$b_{150}$ & $-0,5433906324$ & $b_{151}$ & $-0,5434910728$ & $b_{152}$ & $-0,5435905137$ \\
\hline$b_{153}$ & $-0,5436889722$ & $b_{154}$ & $-0,5437864645$ & $b_{155}$ & $-0,5438830066$ \\
\hline
\end{tabular}

Si on cherche alors les $b_{n}$ sous la forme $b_{n}=C+a / \sqrt{n}$ et $b_{n}=C+a / \sqrt[3]{n}$, on obtient (en utilisant une formule de linéarisation aux moindres carrés):

$$
\begin{aligned}
b_{n}=-0,5736898877+\frac{0,3710889332}{\sqrt{n}}, & \\
& b_{n}=-0,5891153498+\frac{0,2429519729}{\sqrt[3]{n}} .
\end{aligned}
$$

Et la constante calculée ci-dessus vaut

$$
-0,5813148764 \text {. }
$$

On constate donc que la constante calculée est compatible avec les résultats numériques, mais que les «termes correctifs» du développement des $a_{n}$ restent non négligeables. 
Il est aussi possible d'estimer directement la valeur de $b=\alpha^{+}-\alpha^{-}=$ $2 \Im\left(\alpha^{+}\right)$en cherchant les valeurs numériques de $\alpha^{+}$pour différentes valeurs de $\varepsilon$.

On intègre numériquement, pour certains $\varepsilon$ raisonnablement petits, l'équation différentielle $(\mathrm{E})$, par exemple d'une part le long du chemin comprenant les segments $[-1+10 i, 0]$ puis $[0,1]$ et d'autre part le long de $[9,1]$. Ces deux chemins ont la propriété de descendre le relief (cf. fig. 5, et [3]), c'est-à-dire qu'une erreur dans l'estimation des valeurs initiales (en $-1+10 i$ et en 9) devient exponentionnellement petite une fois arrivés en 1 ; on prendra donc les valeurs $v(-1+10 i)=i / 10$ et $v(9)=-1 / 10$. De plus, un de ces chemins part de la montagne Nord, l'autre de la montagne Est, et la définition de $\alpha^{+}$ est qu'il s'agit de la valeur de $\alpha$ pour laquelle il existe une solution tendant vers 0 en l' $\infty$ sur ces deux montagnes (N.B.: on retrouvera la manière de construire les $\alpha$ dans la partie suivante), ce qui sera vrai si et seulement si les deux $v(1)$ trouvés sont égaux.

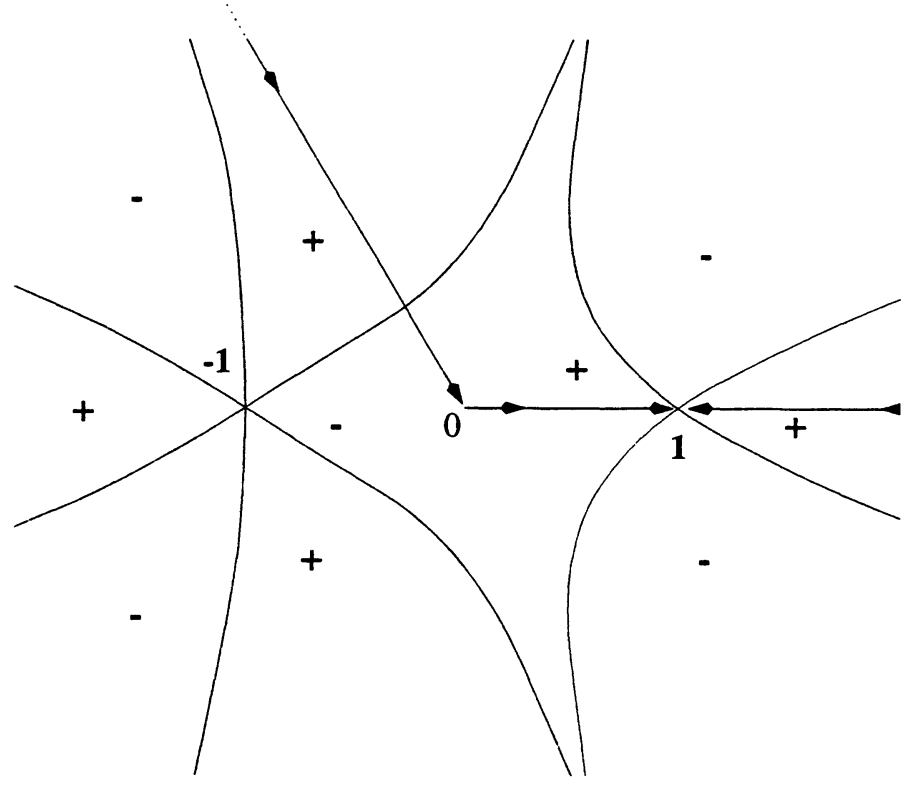

Fig. 5 Relief pour l'équation de Van der Pol, et chemins pour l'intégration numérique 


\section{Éric Matzinger}

Une recherche numérique de ce type donne les approximations suivantes:

\begin{tabular}{|c|l|c|}
\hline$\varepsilon$ & \multicolumn{1}{|c|}{$\alpha^{+}$} & $2 \Im\left(\alpha^{+}\right) \times e^{4 / 3 \varepsilon} \sqrt{\varepsilon}$ \\
\hline \hline 0,20 & $0,9684+0,00153 i$ & 1,07 \\
\hline 0,17 & $0,9733+0.00055 i$ & 1,16 \\
\hline 0,14 & $0,9800+0.000120 i$ & 1,23 \\
\hline 0,08 & $0,9893+1,40 \cdot 10^{-7} i$ & 1,37 \\
\hline 0,06 & $0,9921+6,48 \cdot 10^{-10} i$ & 1,42 \\
\hline 0,05 & $0,9935+8,5 \cdot 10^{-12} i$ & 1,44 \\
\hline 0,04 & $0,9948+1,23 \cdot 10^{-14} i$ & 1,47 \\
\hline
\end{tabular}

Ces résultats sont compatibles avec la valeur de la constante trouvée (égale environ à 1,68 ), avec des termes correctifs en $\sqrt{\varepsilon}$ et $\sqrt[3]{\varepsilon}$.

\section{Bibliographie}

[1] Benoît (Éric), Callot (Jean-Louis), Diener (Francine) et Diener (Marc). Chasse au canard, Collectanea Mathematica, 31(1-3), 37-119, 1981.

[2] Canalis-Durand (Mireille). - Caractère Gevrey du développement formel des solutions canard de l'équation de van der Pol, C.R. Acad. Sciences Paris Sér. I Math., 311 (1), 27-30, 1990.

[3] FRUChARD (Augustin) et SCHÄFKE (Reinhard). - Exceptional complex solutions of the forced Van der Pol equation, Funkcialaj Ekvacioj, 42(2), 201-223, 1999.

[4] LiÉNARD (A.). - Étude des oscillations entretenues, Revue générale d'électricité, 22(23), 901-954, 1928.

[5] Olver (F.W.J.). - Asymptotics and special functions, New York, Academic Press, 1974.

[6] VAn Der Pol. - Relaxation oscillations, Philos. Mag., (2-7), 978-992, 1926. 